Revue des patrimoines

\title{
La famille Richou: de la meunerie à la banque en Anjou (fin XVIIIe siècle-début XXe siècle)
}

\section{Claire Steimer et Viviane Manase}

\section{(2) OpenEdition}

\section{Journals}

Édition électronique

URL : http://journals.openedition.org/insitu/3443

DOI : 10.4000/insitu.3443

ISSN : $1630-7305$

Éditeur

Ministère de la culture

Référence électronique

Claire Steimer et Viviane Manase, "La famille Richou : de la meunerie à la banque en Anjou (fin XVIIIe siècle-début XXe siècle) », In Situ [En ligne], 8 | 2007, mis en ligne le 01 mars 2007, consulté le 19 avril 2019. URL : http://journals.openedition.org/insitu/3443; DOI : 10.4000/insitu.3443

Ce document a été généré automatiquement le 19 avril 2019

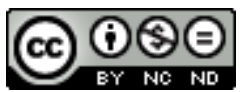

In Situ Revues des patrimoines est mis à disposition selon les termes de la licence Creative Commons Attribution - Pas d'Utilisation Commerciale - Pas de Modification 4.0 International. 


\title{
La famille Richou : de la meunerie à la banque en Anjou (fin XVIIIe siècle- début XXe siècle)
}

\author{
Claire Steimer et Viviane Manase
}

1 À l'instar du reste de la France, l'Anjou connaît au cours du XIXe siècle un vaste mouvement de modernisation agricole et de développement industriel (fig. $\mathbf{n}^{\circ} \mathbf{1}$ ). La mise en place de techniques d'élevage et de culture novatrices suscite un nouveau mode d'organisation des exploitations et des fermes. On assiste alors à une période de reconstruction ou de réaménagement des bâtiments qui vise à rationaliser l'espace rural, à appliquer les principes hygiénistes et à accroître la productivité. 


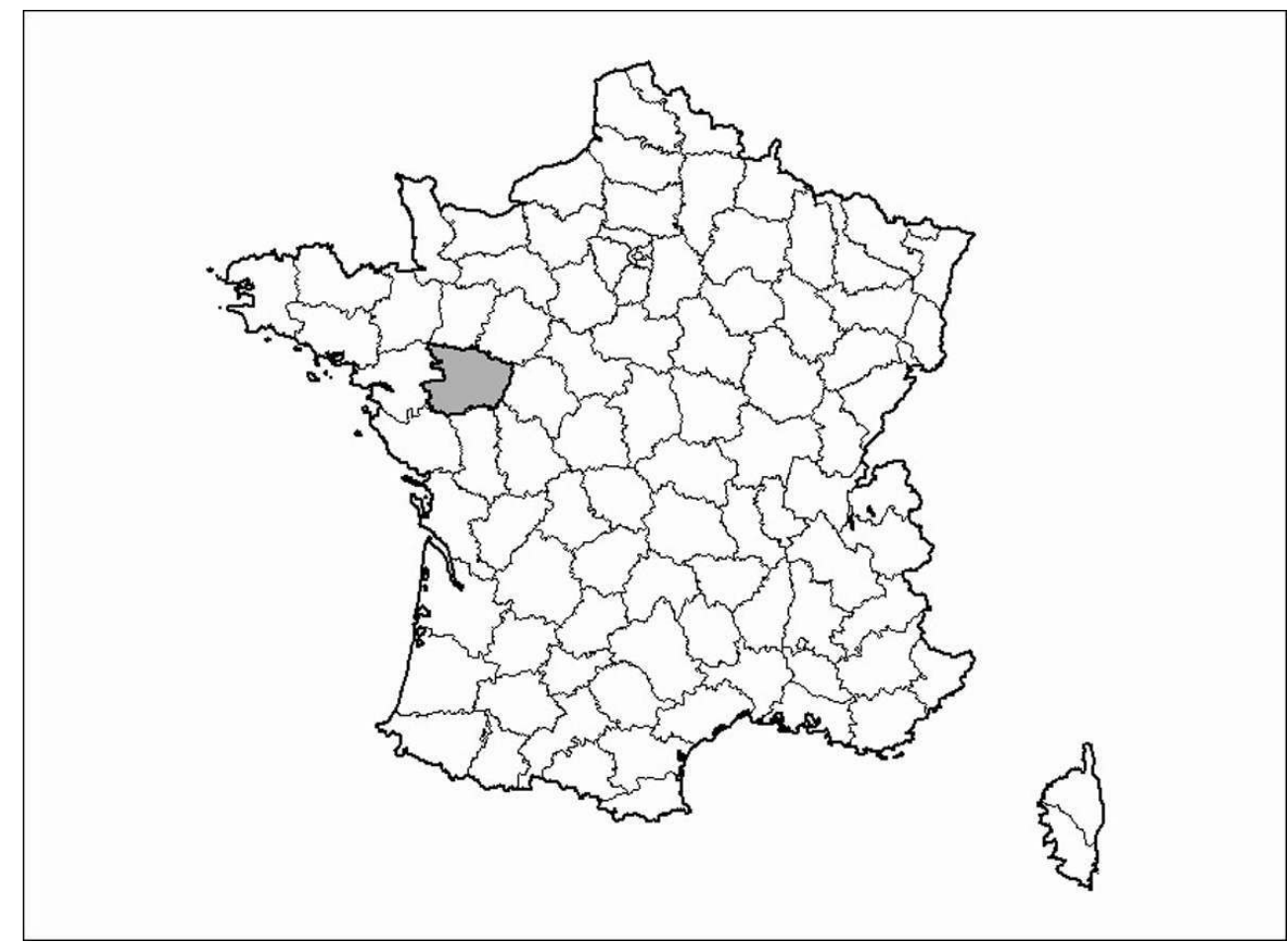

Carte de situation du département de Maine-et-Loire. Source : Conseil général. Service géomatique (c) Conseil général de Maine-et-Loire, 2006.

2 L'étude de quelques fermes de la deuxième moitié du XIXe siècle du canton du Liond'Angers $^{1}$ évoque concrètement cette mutation agricole (fig. $\mathbf{n}^{\circ} 2$ ). Propriétés d'une même famille, les Richou, elles constituent un témoignage intéressant, point de départ d'un parallèle entre l'évolution des biens fonciers et, de façon plus large, l'histoire économique et sociale de cette famille. Jusque-là plutôt associée à l'histoire bancaire angevine - la banque Richou est créée à Angers en 1827 - c'est en fait la meunerie qui est, semble-t-il, à l'origine de l'ascension sociale de cette famille, confortée par l'exploitation de biens agricoles. Si les membres de la noblesse ont, après la Révolution, repris en partie possession de leurs terres et réaffirmé leur pouvoir social affaibli, la bourgeoisie issue du négoce, de l'industrie ou des finances compose désormais une puissante classe de notables. Le modèle aristocratique reste pourtant prégnant pour ces familles en quête de reconnaissance sociale: preuve en est l'arbre généalogique que Raymond Richou fait réaliser en 1922, accompagné d'une recherche d'armoiries². 
PAYS DU HAUT-ANJOU SEGREEN

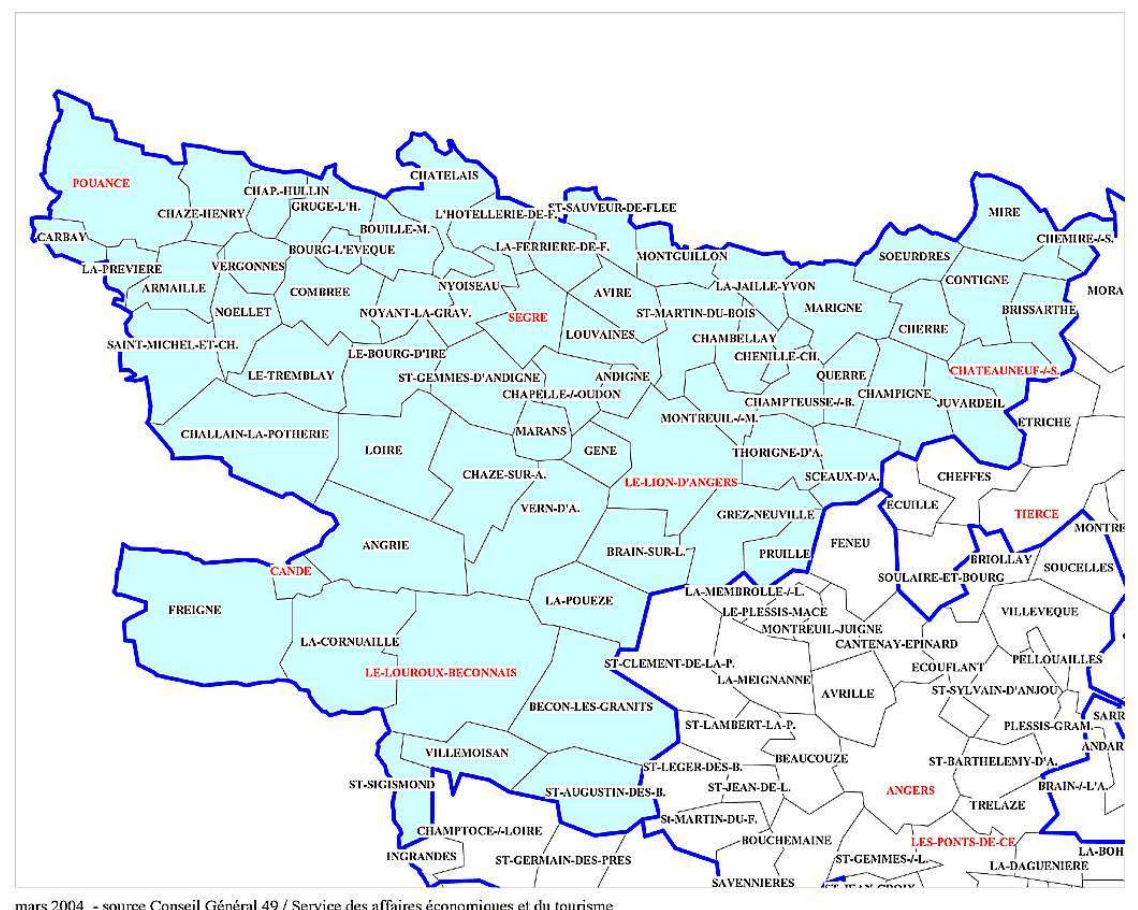

Carte du Pays du Haut-Anjou segréen. Source : Conseil général. Service des affaires économiques et du tourisme, 2004

(c) Conseil général de Maine-et-Loire, 2006.

Il ne s'agit pas ici de recenser l'ensemble des membres de la famille ni leurs propriétés, mais de présenter un panorama des activités d'une famille bourgeoise au cours d'un siècle marqué par la révolution agricole et industrielle, dans une région dominée par la grande propriété terrienne d'origine aristocratique.

\section{Les Richou, minotiers}

\section{Le moulin de la Roussière : berceau de la famille}

D'après les sources consultées, la mention la plus ancienne des Richou date de 1747 et établit le lien manifeste de cette famille avec la meunerie: le sieur Hullin de la Coudre vend à Pierre Richoust (sic), meunier, et Renée Soreau sa femme, une métairie à Feneu ${ }^{3}$. Le couple est alors installé à La Membrolle-sur-Longuenée, au moulin de la Roussière, qui constitue le berceau et le point d'ancrage de la famille pendant plus d'un siècle ${ }^{4}$.

L'importance économique des moulins est ancienne, la force hydraulique étant la première à être utilisée ${ }^{5}$. Ce sont les seuls ateliers de transformation ou d'affinage de nombreuses matières premières : sciage du bois et de la pierre, battage des peaux, des textiles et des métaux, broyage des graines oléagineuses et du tan. Dans les limites actuelles du département de Maine-et-Loire, on dénombre sur la carte de Cassini (1763-1769) 723 moulins à vent et 634 moulins à eau, tandis que le recensement impérial de 1809 en donne un total respectivement de 912 et 613. Avant la Révolution, ces moulins 
appartenaient principalement aux familles nobles et aux communautés religieuses. Le moulin de la Roussière dépendait ainsi d'un ancien fief et seigneurie avec château-fort, dont une tour subsistait au début du XVIe siècle, sur une haute motte dominant la rive droite de la Mayenne. Un aveu de 1408 atteste l'existence des moulins, porte, portineau et chaussée situés sur la rivière de la Mayenne, près de l'ancien château de la Roussière entouré de douves. Transformée en simple métairie et rattachée en 1499 au château du Plessis-Macé, La Roussière est ainsi décrite dans un autre aveu : « la place du vieil chastel du dit lieu de la Roussière avec une motte en laquelle a encore partie d'une tour ancienne et [...] un moulin à tan et aussi la place d'une maison et jardins qui étaient dans la baillée d'iceluy moulin à tan [...]. La maison de [mes] moulins du dit lieu de la Roussière, en laquelle a trois moulins tous à blés avec la chaussée, porte et portineau [...]. Une autre maison sise près [mes] dits moulins en laquelle demeurent les mouliniers d'iceulx avec l'arrière et des charges des dits moulins $»^{6}$. Puis l'hôtel-Dieu d'Angers en devient au XVIIIe siècle propriétaire et fait reconstruire les moulins en 1758, pour finalement les vendre en 1780 au comte de Serrant, propriétaire du Plessis-Macé. Instruments des seigneurs et des puissantes communautés religieuses, la population était obligée d'y moudre son grain contre redevance : la symbolique seigneuriale liée aux moulins était donc particulièrement forte.

6 Au début du XVIIIe siècle, Mathurin Soreau († 1707) puis son fils François (1680-1764) y sont meuniers. Les Richou sont alors associés à l'exploitation du moulin, par le mariage, en 1732, de Pierre Richou (1710-1779) et Renée Soreau (1711-1771), fille de François. Ils restent simples exploitants jusqu'en 1811, époque à laquelle René (II) Richou (1774-1840) acquiert le moulin de la Roussière appartenant au comte de Serrant. C'est ainsi qu'un simple meunier parvient à racheter l'un des symboles du pouvoir seigneurial à la puissante famille Walsh de Serrant.

7 Les Richou se donnent tous les moyens pour développer la productivité du moulin et participent ainsi à la vague de modernisation que connaît la meunerie traditionnelle au XIXe siècle: c'est l'époque des grandes minoteries, utilisant des roues de dessous et surtout des turbines très puissantes qui améliorent le rendement et conduisent à la ruine des petits moulins. Ce sont les deux fils de René (II), Alexandre (I) (1809-1888), maire de La Membrolle et Auguste (1811-1878), marchand de fer, tous deux minotiers associés, qui sont les acteurs de ces transformations à la Roussière : ils y construisent en 1847 une importante minoterie avec une chute d'eau d'une hauteur constante de $90 \mathrm{~cm}$, qui fait mouvoir deux roues, et une machine à vapeur verticale, système Powell, d'une force de 20 chevaux $^{7}$. Ces deux moteurs agissent alors sur 13 paires de meules ${ }^{8}$ (fig. $\mathbf{n}^{\circ} \mathbf{3}$ ). 
Figure 3

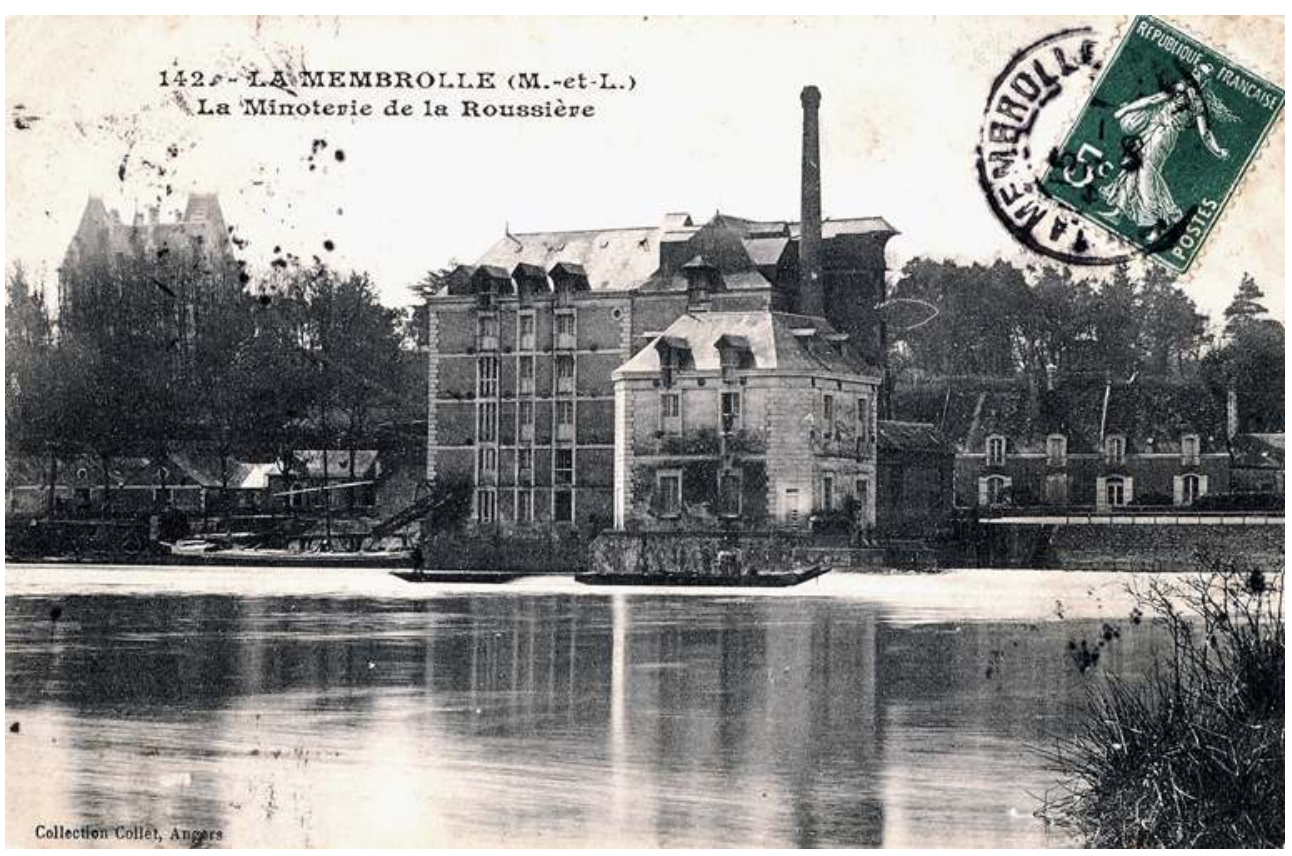

La minoterie de la Roussière, carte postale, A.D. Maine-et-Loire, 6 Fi. Rousseau, Bruno

(c) ADAGP/Conseil général de Maine-et-Loire, 2004.

8 En outre, on note la réalisation de plusieurs agrandissements qui témoignent du développement et de la modernisation de l'usine9. Un acte du 29 juillet 1872, par lequel Alexandre (I) cède la Roussière à ses fils - Alexandre (II) (1835-1897) et René-Eugène (1841-1900) - en fournit une description qui permet de mesurer l'importance du site de production avec zones de stockage et de préparation pour le transport: "l'usine dite moulin à eau avec la chute d'eau [...], les magasins à charbon, actuellement entourés de murs établis sur le lieudit le pâtis, la boulangerie de la maison de maître de la Roussière, les bureaux qui sont au-dessus, les magasins à blé et à farine bâtis sur l'emplacement de l'ancienne butte de vidange qui est au levant du jardin mais non compris les toits à porcs existant en ce bâtiment et un magasin à sacs nouvellement construit sur une parcelle de terrain appelée le Port au levant des magasins à blé et à farine ci-dessus désignés ; ainsi que la maison de maître de la Roussière avec servitudes et dépendances; la maison du jardinier et celle du contremaître de l'usine et les terres et les prés les environnant $»^{10}$ (fig. $\left.n^{\circ} 4\right)$.

9 Les démarches pour perfectionner la minoterie se succèdent: en 1875 , les deux frères souhaitent élargir les coursiers pour y installer des roues perfectionnées afin d'augmenter leur force motrice hydraulique ${ }^{11}$ (fig. $\mathbf{n}^{\circ}$ 5) et en octobre 1883, ils demandent l'autorisation d'élever un bâtiment au-dessus de la nouvelle roue hydraulique qu'ils viennent d'établir ${ }^{12}$. 


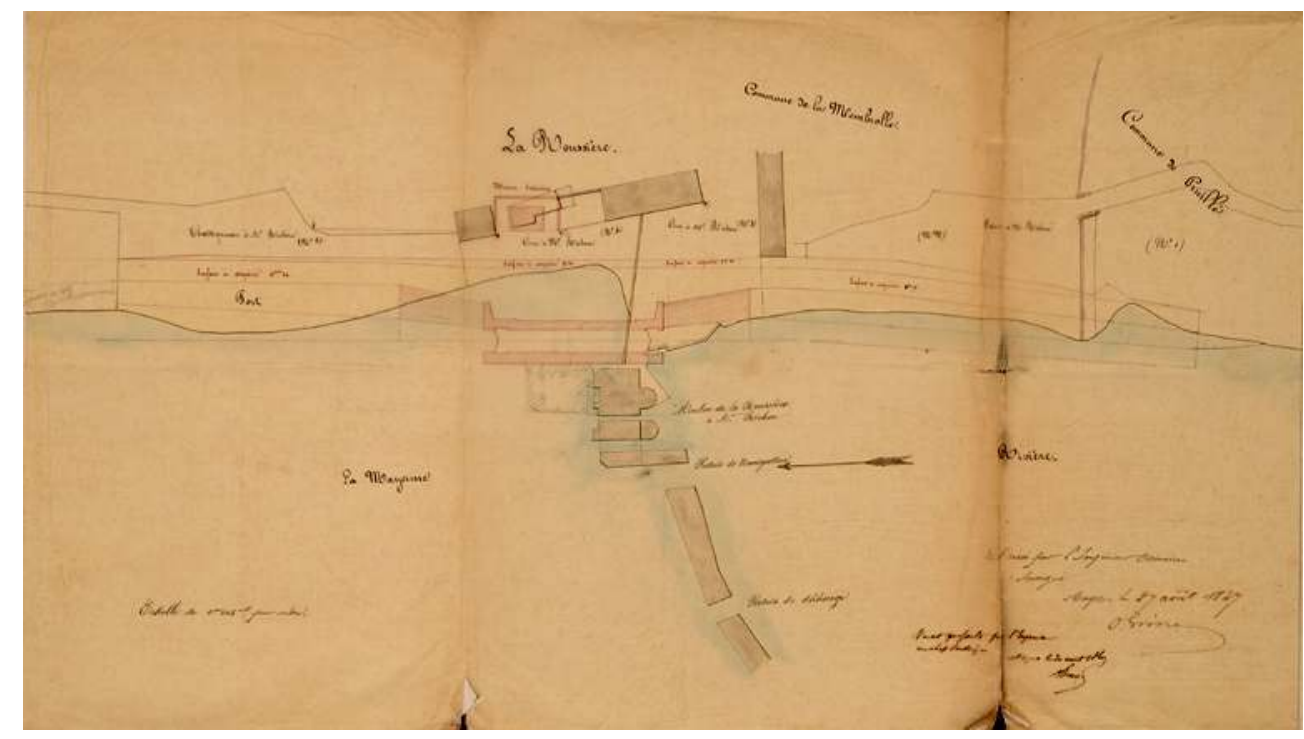

Plan terrier dressé par l'ingénieur ordinaire le 27 août 1847, construction de l'écluse de la Roussière, A.D. Maine-et-Loire, 126 S 12. Rousseau, Bruno

(c) Conseil général de Maine-et-Loire, 2006.

Figure 5

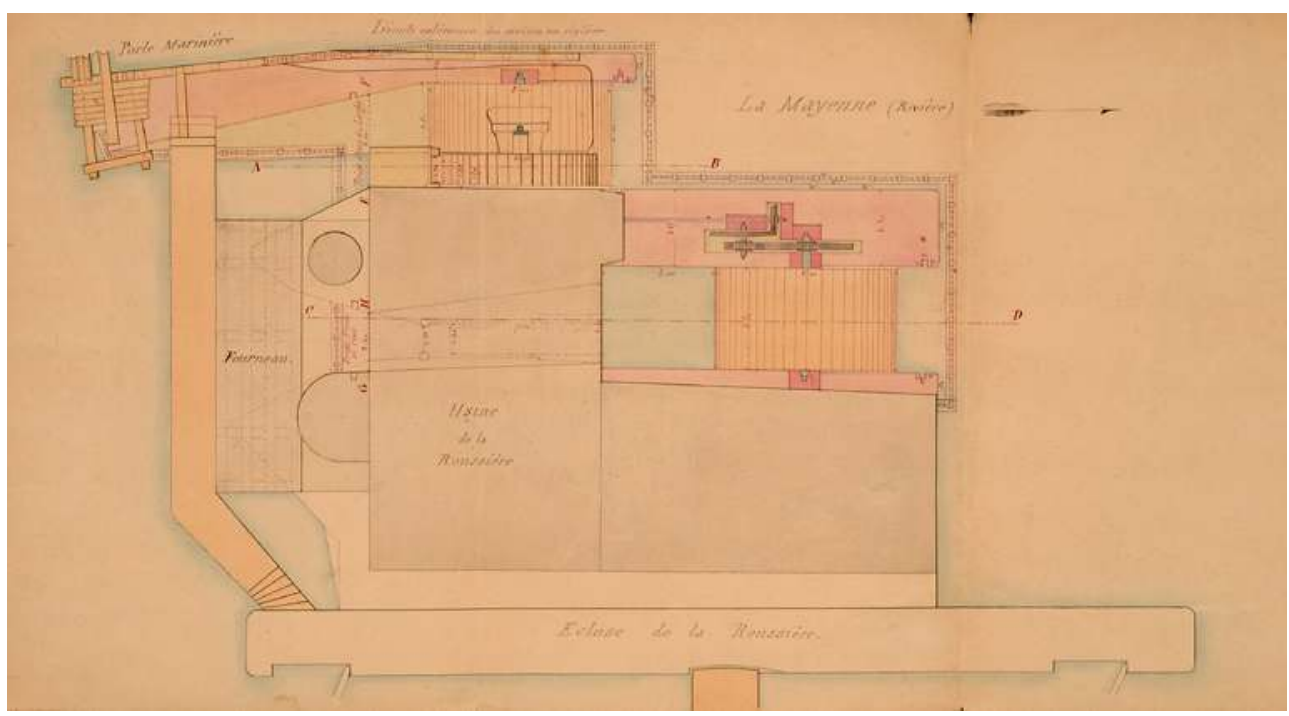

Modification des coursiers de l'usine de la Roussière : plan de détail dressé par l'ingénieur ordinaire le 30 novembre 1875, A.D. Maine-et-Loire, 126 S 12. Rousseau, Bruno

(c) Conseil général de Maine-et-Loire, 2006.

10 La fin du XIXe siècle voit la poursuite de ce développement : le bâtiment est reconstruit dans les années 1894-1904, un magasin est ajouté entre 1897 et 1904, mais en 1919, le moulin est accidentellement incendié ${ }^{13}$. Reconstruit et encore en fonction en 1936 avec une puissance de 16 chevaux, il est définitivement abandonné en 1942, et suit en cela le déclin général des moulins à eau : si en 1862 il y a encore 609 usines hydrauliques en Maine-et-Loire, il n'en subsiste que 131 en 1946. 
De la fin du XVIIIe siècle au début du XXe siècle, la Roussière est restée aux mains des Richou, qui y ont concentré tous leurs efforts et en ont fait un exemple de réussite de la minoterie industrielle. Berceau de la famille, la prospérité et l'importance de ce lieu pour leurs propriétaires apparaissent à travers l'élégant château édifié sur les hauteurs dominant la Mayenne. En 1854, la maison du Rocher et celle de la Roussière sont élevées, remplacées en 1901 par le château du Petit Rocher ${ }^{14}$ (fig. $\mathbf{n}^{\circ}$ 6).

Figure 6

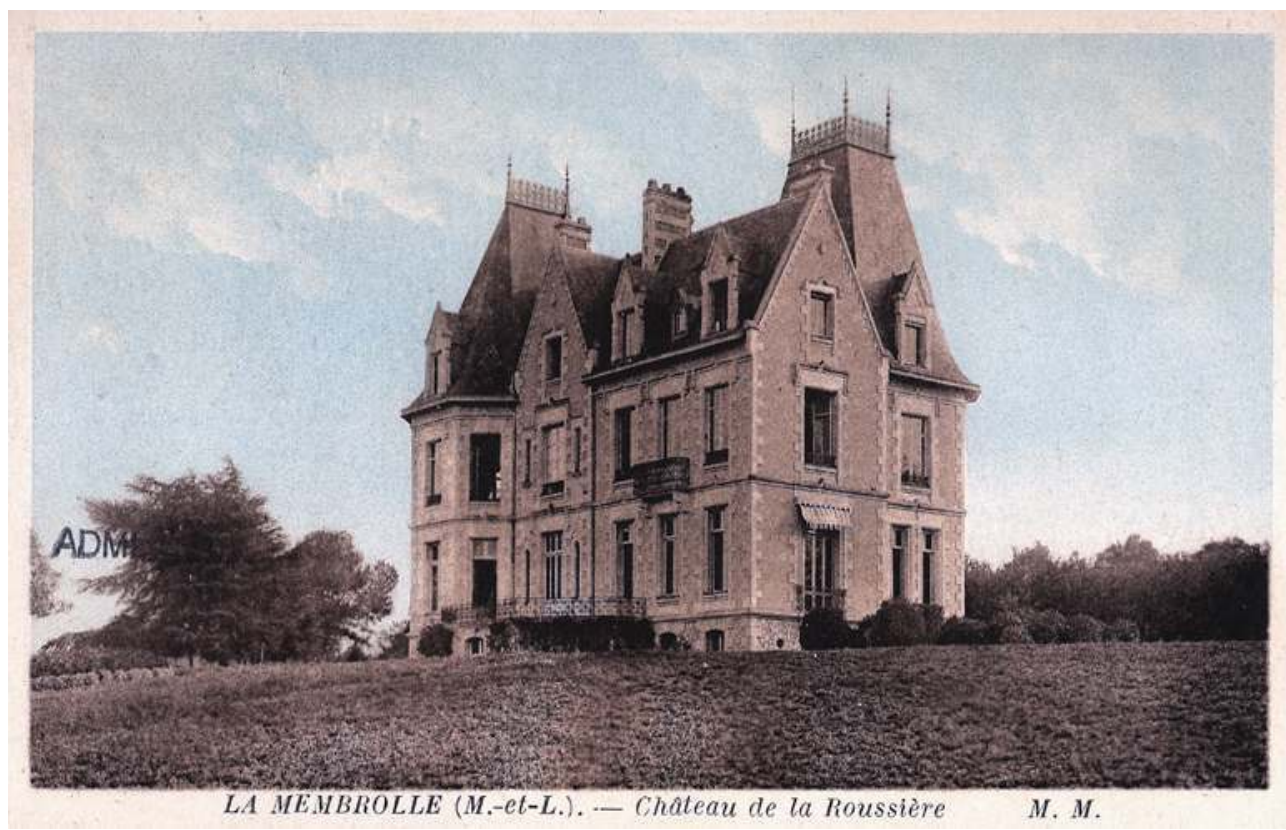

Le château de la Roussière, carte postale. A.D. Maine-et-Loire, 6 Fi. Rousseau, Bruno

(c) Conseil général de Maine-et-Loire, 2006.

\section{Moulins à vent et à eau : une affaire de famille}

Si le moulin de la Roussière constitue le cœur de la branche Richou-Dugré, d'autres moulins sont également aux mains de la branche Richou-Guilleux. Il s'agit aussi bien de moulins à vent que de moulins à eau, installés sur les bords de la Mayenne et de la Sarthe. Dans un premier temps, la meunerie à vent a été moins touchée par la crise que la meunerie traditionnelle à eau: dynamisée entre 1850 et 1870 par l'adoption des ailes Berton, elle ne tarde pourtant pas à péricliter.

Les deux types de meunerie ne sont pas incompatibles : un même propriétaire pouvait à la fois profiter d'un moulin à eau et d'un moulin à vent, exploitant en alternance les vents les plus forts et le débit maximum de l'eau. C'est ainsi que Pierre Richou (né en 1767) et Rosalie Ménard, installés aux moulins à eau du Petit Charay et du Grand Charay à Chambellay, achètent en mars 1832 le moulin à vent de la Rocherie à Châteauneuf-surSarthe pour 2500 francs : il s'agit d'un moulin de type chandelier, entièrement en bois, couvert d'ardoises, ayant deux harnois ${ }^{15}$. Élevé sur le coteau de la Rocherie par un certain René Jacques Dagonneau sur un terrain acquis en 1817, il comporte un magasin surmonté d'un grenier, et un appentis à usage de remise ${ }^{16}$. Il est rapidement revendu, avec une maison, en février 1843, à François Lorieux, meunier demeurant au moulin de Charnacé (Champigné) $^{17}$. 


\section{(fig. $\mathbf{n}^{\circ}$ 7).}

Figure 7

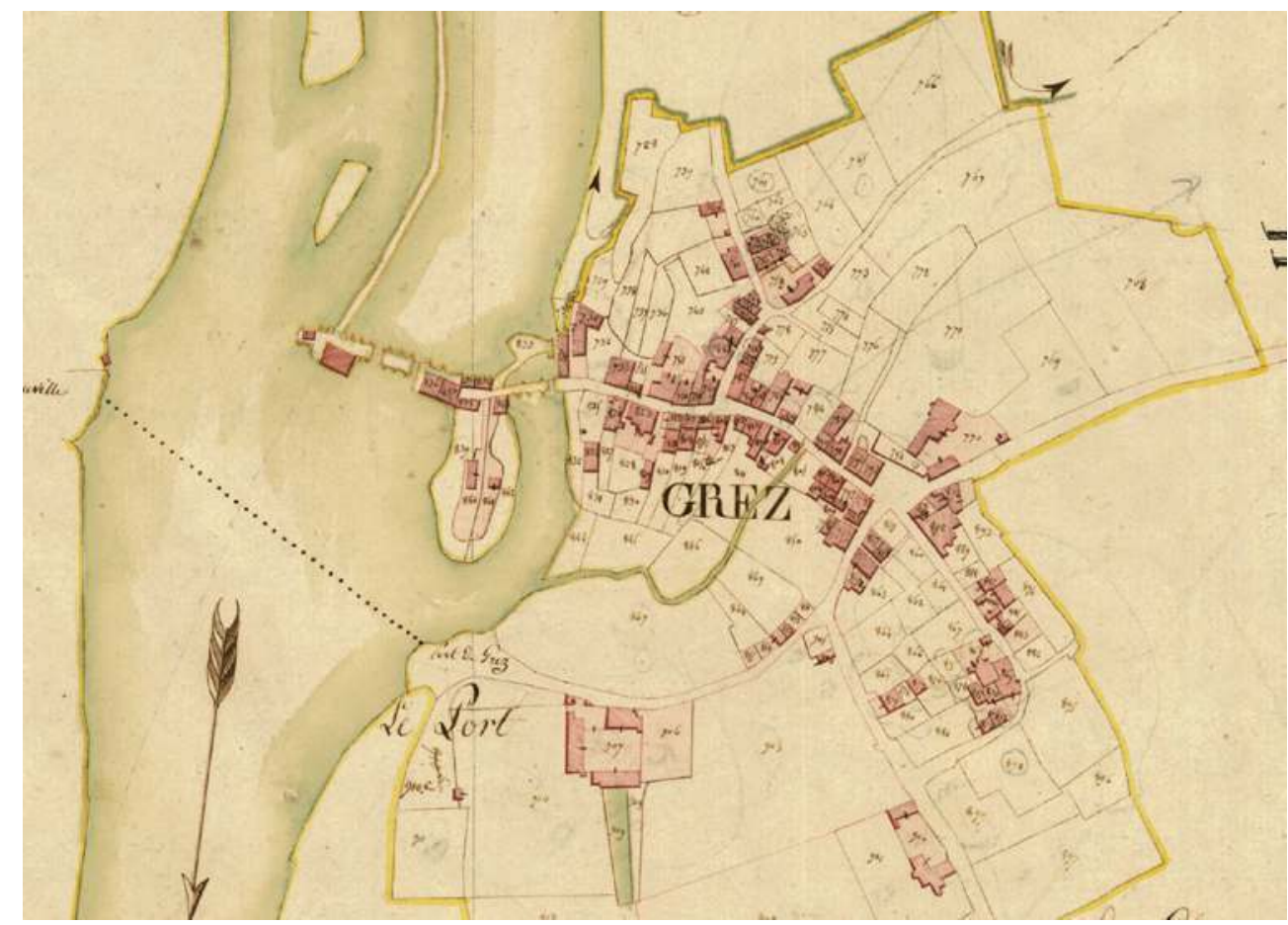

Moulin à eau de Grez-Neuville, cadastre napoléonien, 1810. A.D. Maine-et-Loire. Rousseau, Bruno (c) Conseil général de Maine-et-Loire, 2005

Il l'avait acquis peu de temps auparavant (en 1800) des enfants du meunier Joseph Guilleux. Il était alors composé d'« un moulin à deux roues dont une à double harnois, un logement pour le fermier, un grand grenier, une écurie dans le bourg de Neuville, chambre à côté, grenier sur le tout, une étable à vaches, les anciens ponts de Neuville 
composés d'une ancienne arche, de différents piliers, des débris des dits ponts sur laquelle arche est une loge, deux toits à porcs, un jardin d'environ 12 ares ou 2 boissellées [...], 5 motterons dont deux adjacents à la chaussée et trois petits au milieu des eaux nommés les rutors ». Ce moulin vendu très rapidement par les Richou sera, comme la Roussière, reconstruit dans la deuxième moitié du XIXe siècle et modernisé par une autre famille de meuniers.

Les répertoires alphabétiques des registres des hypothèques attestent également de l'existence de Richou meuniers, tout particulièrement à la minoterie de Cheffes-surSarthe ${ }^{21}$, mais aussi dans la région de Mozé-sur-Louet, au Mesnil à Saint-Martin-du-Bois et à Cheviré-le-Rouge. L'activité meunière des Richou s'étend ainsi beaucoup plus largement en Anjou et leur donne une position singulière dans la société angevine de l'époque. Le meunier était en effet l'artisan incontournable de la phase de transformation des céréales, base essentielle de l'alimentation. Sa maîtrise des éléments, eau et vent, ses compétences techniques, la monumentalité et la complexité de sa machine, renforçaient encore le respect qu'on lui vouait ${ }^{22}$. Les Richou ont su mettre à profit ce statut pour s'élever dans l'échelle sociale en diversifiant très rapidement leurs activités. Ils accumulent ainsi nombre de biens fonciers et se constituent un patrimoine confortable. Les importants investissements dans les terres agricoles, à un moment où leur rentabilité et leur productivité s'accroissent nettement, constituent un moyen supplémentaire d'asseoir un statut social déjà reconnu dans le monde rural.

\section{Des propriétaires fonciers dans le Haut-Anjou segréen}

\section{Une forte implantation territoriale}

20 Le Haut-Anjou segréen est une région où la révolution agricole a été fortement relayée par de grandes figures comme le comte de Falloux qui, dans sa ferme modèle de Bourgd'Iré, met en application les nouvelles normes agricoles destinées à accroître la productivité des domaines. Les Richou, à leur échelle, ont participé à ce mouvement de modernisation. Difficile d'étudier l'ensemble du domaine foncier de cette vaste famille: l'acte de donation entre vifs d'Anne Dugré (1748-1825), veuve de René (I) Richou (1745-1807), daté du 23 octobre 1823, permet cependant d'en mesurer l'importance ${ }^{23}$. Il s'agit de terres situées dans la commune du Lion-d'Angers et des environs, léguées par ses parents, Julien Dugré et Marie-Anne Richou. Anne Dugré a également acquis une partie de ces terres au cours de son mariage et après la mort de René (I) en 1807. Ce sont bien souvent de grands noms de la noblesse angevine - le comte Walsh de Serrant, Mme de Meaulne - qui ont vendu ces biens. Les Richou ont ainsi, semble-t-il, bénéficié des difficultés rencontrées par ces grandes familles au lendemain de la Révolution. Cet acte de donation fait état de 17 métairies, dont la surface de terres labourables varie entre 20 et 39 hectares, et une dizaine de closeries de 3 à 7 hectares. Elles sont exploitées par des fermiers selon les règles fixées par le bail, le principe de l'exploitation à moitié fruit étant fréquemment rencontré. Le revenu d'une closerie oscille entre 140 et 560 francs, celui d'une métairie est d'au moins 700 francs et peut atteindre 2370 francs. Si cet acte de donation permet de fournir quelques repères, il ne rend toutefois pas compte de l'ensemble des propriétés détenues au cours du XIXe siècle par les Richou en Anjou (fig. $\mathbf{n}$ $\circ 8)$. 


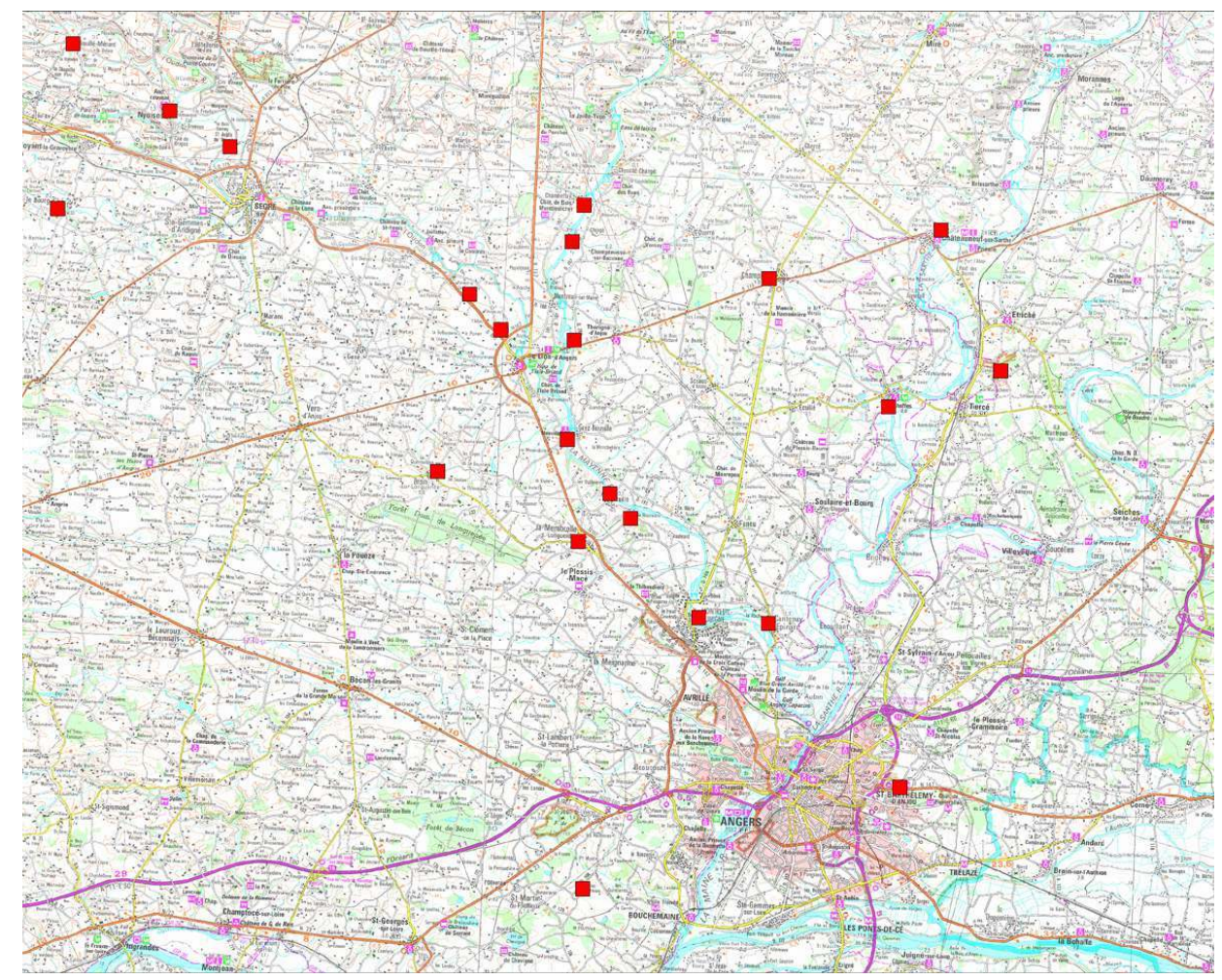

Carte IGN de situation au $100000^{e}$ : principales propriétés des Richou. Steimer, Claire

(c) Conseil général de Maine-et-Loire, 2006.

\section{(Re)construction et organisation des fermes}

21 L'étude de quelques fermes appartenant aux fils de René (II) permet d'en apprécier l'organisation et le fonctionnement. Reconstruites ou réaménagées dans la deuxième moitié du XIXe siècle suivant les schémas préconisés par la révolution agricole, ces fermes témoignent d'une rationalisation des espaces et d'un souci d'hygiène pour une meilleure productivité. Si dans certains cas la reconstruction est totale, bien souvent une partie des bâtiments plus anciens est conservée, les bâtiments de dépendance modernes étant ajoutés à proximité. Bien qu'il n'y ait réellement ni modèle ni plan spécifique, les nouvelles constructions prenant en compte les bâtiments existants, on note toutefois un effort d'organisation des bâtiments autour de la cour et une spécialisation selon leurs fonctions. Généralement, le logis reconstruit pour répondre aux nouvelles exigences de confort se distingue par le soin apporté à sa façade ordonnancée. Quant aux matériaux, l'usage de la brique pour les encadrements de baies est caractéristique de cette époque de construction et largement répandu tant pour les dépendances que pour le logis.

Transmise par René (II) à ses fils, la Brissetière (Le Lion-d'Angers) est composée à l'origine d'un seul corps de bâtiment avec un petit logis du XVIe siècle et des dépendances du XVIIIe siècle (fig. $\mathbf{n}^{\circ} \mathbf{9}$ ). 
Figure 9

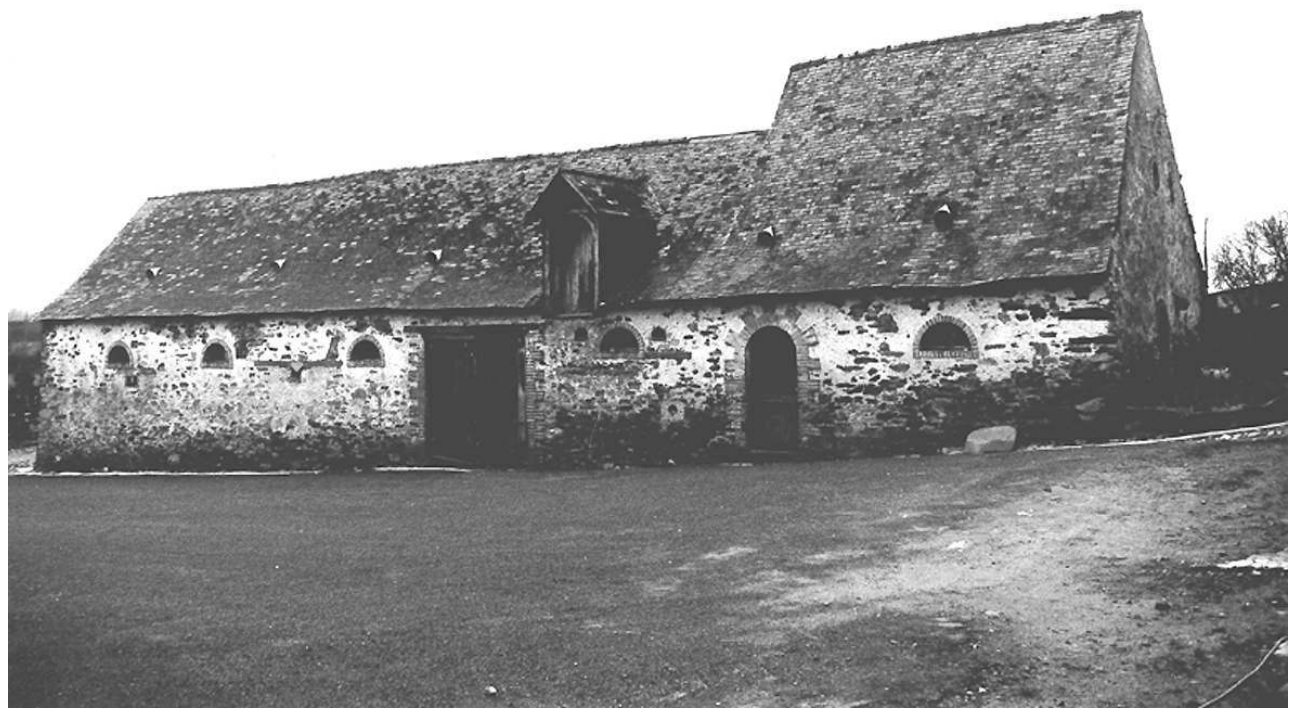

La Brissetière (Le Lion-d'Angers), ancien logis transformé en dépendance. Manase, Viviane (c) ADAGP/Conseil général de Maine-et-Loire, 1999.

Encore visible, ce bâtiment a vraisemblablement été transformé au XIXe siècle en simple

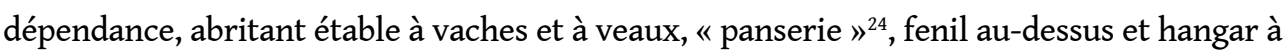
l'arrière. En 1864, Alexandre (I) fait élever un nouveau logis qui se trouve ainsi dissocié des bâtiments de dépendance (fig. $\mathbf{n}^{\circ} \mathbf{1 0}$ ). 


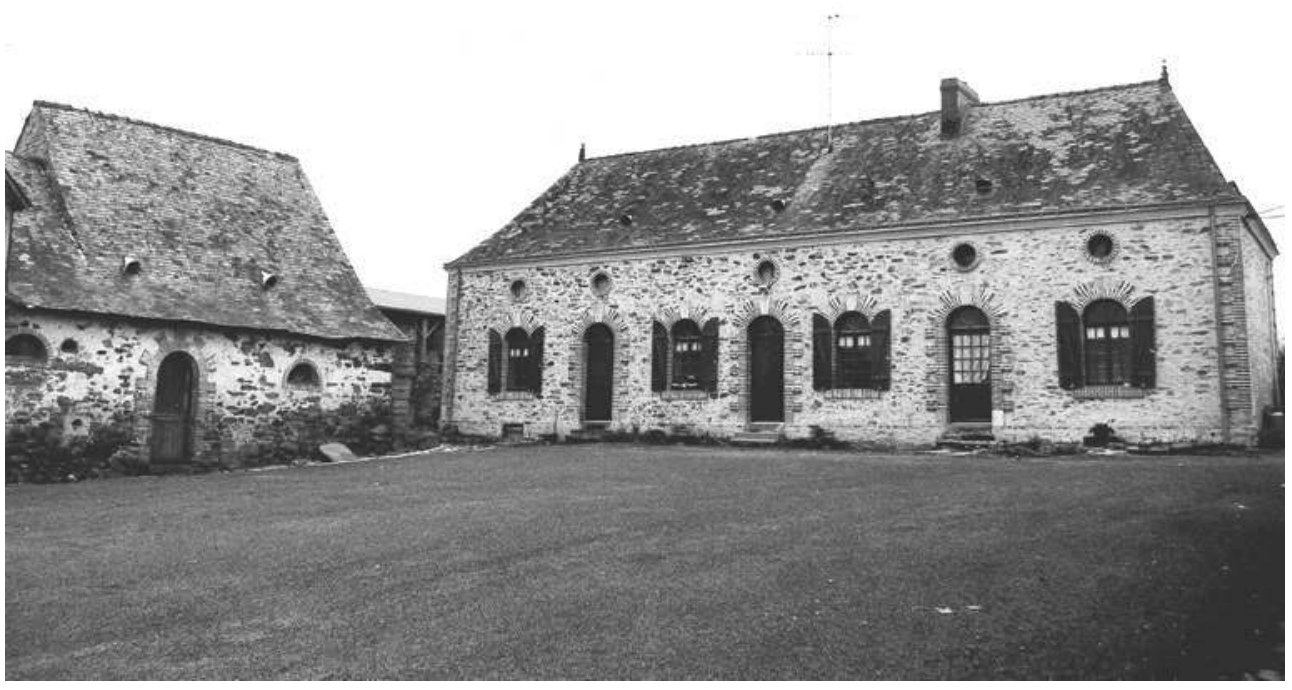

La Brissetière (Le Lion-d'Angers), nouveau logis. Manase, Viviane

(c) ADAGP/Conseil général de Maine-et-Loire, 1999.

C'est en effet une des spécificités de cette époque de construire les bâtiments à distance les uns des autres pour éviter les incendies. Une étable à chevaux et une porcherie surmontées d'un fenil sont aussi ajoutées. Chaque bâtiment a donc une fonction spécifique qui rend l'organisation de la ferme bien plus rationnelle. En 1878, la métairie comprend 29 hectares 47 ares avec un revenu brut de 2700 francs $^{25}$ mais son développement ne s'arrête pas là : à partir de 1881, Arthur (fils d'Auguste, né en 1845) complète cet ensemble par la construction d'une seconde porcherie à l'arrière du logis avec un enclos, un bûcher et des latrines, ainsi qu'une remise à l'ouest. À Chauvon (Le Lion-d'Angers), Auguste fait construire en 1844 de nouveaux bâtiments à côté d'une ancienne ferme détruite en 1850 lors de l'établissement de la nouvelle route. Le plan choisi est régulier, pour rendre le fonctionnement de la ferme plus efficace : le logis et les étables édifiés sur une même ligne (fig. $\mathbf{n}^{\circ} \mathbf{1 1}$ ), la remise-grange à larges et hautes ouvertures cintrées en brique (fig. $\mathbf{n}^{\circ} \mathbf{1 2}$ ) ainsi que la porcherie, donnent sur la cour. 
Figure 11

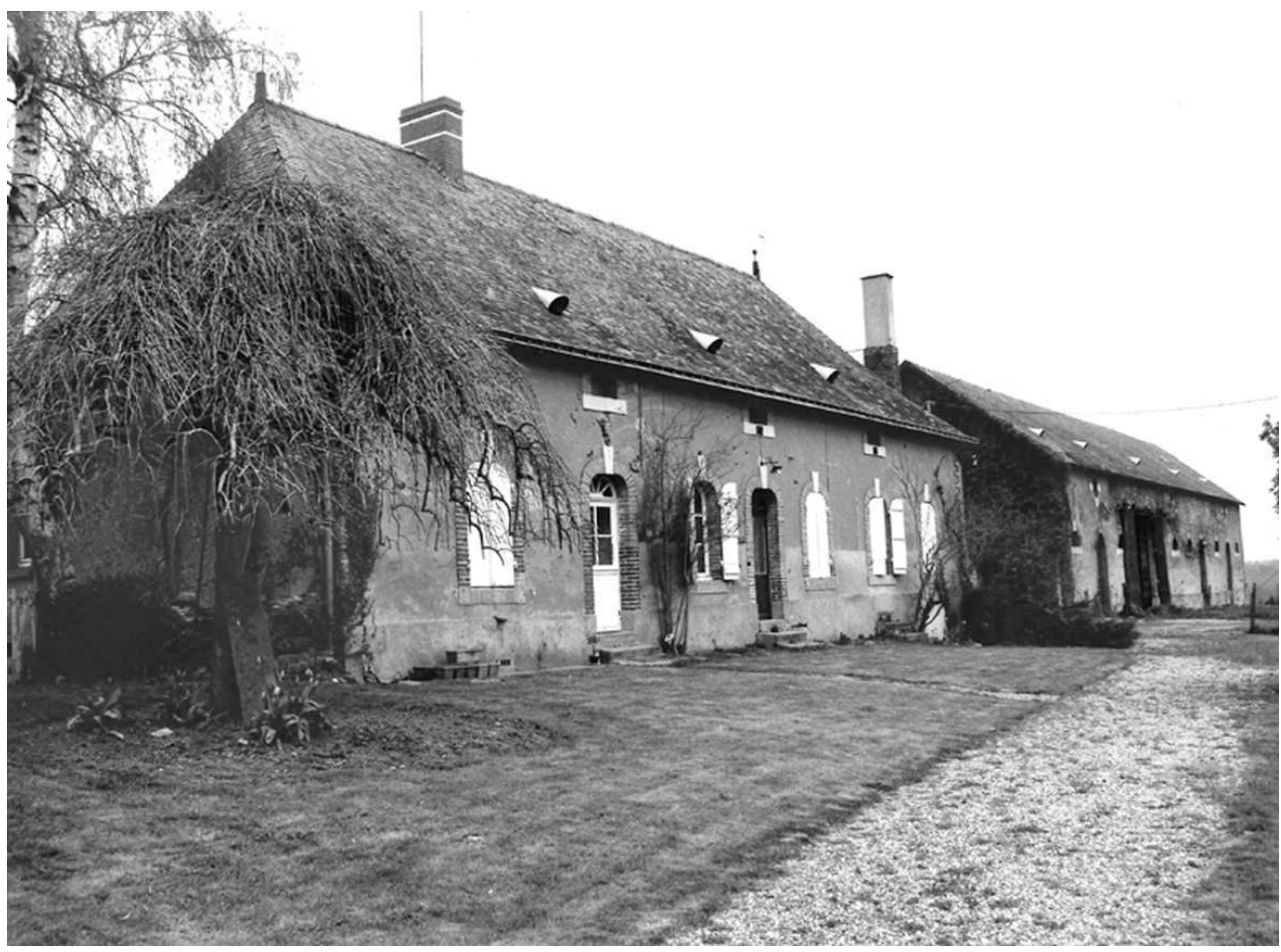

Chauvon (Le Lion-d'Angers), logis et étables dans le prolongement. Manase, Viviane

(c) ADAGP/Conseil général de Maine-et-Loire, 1999.

Figure 12

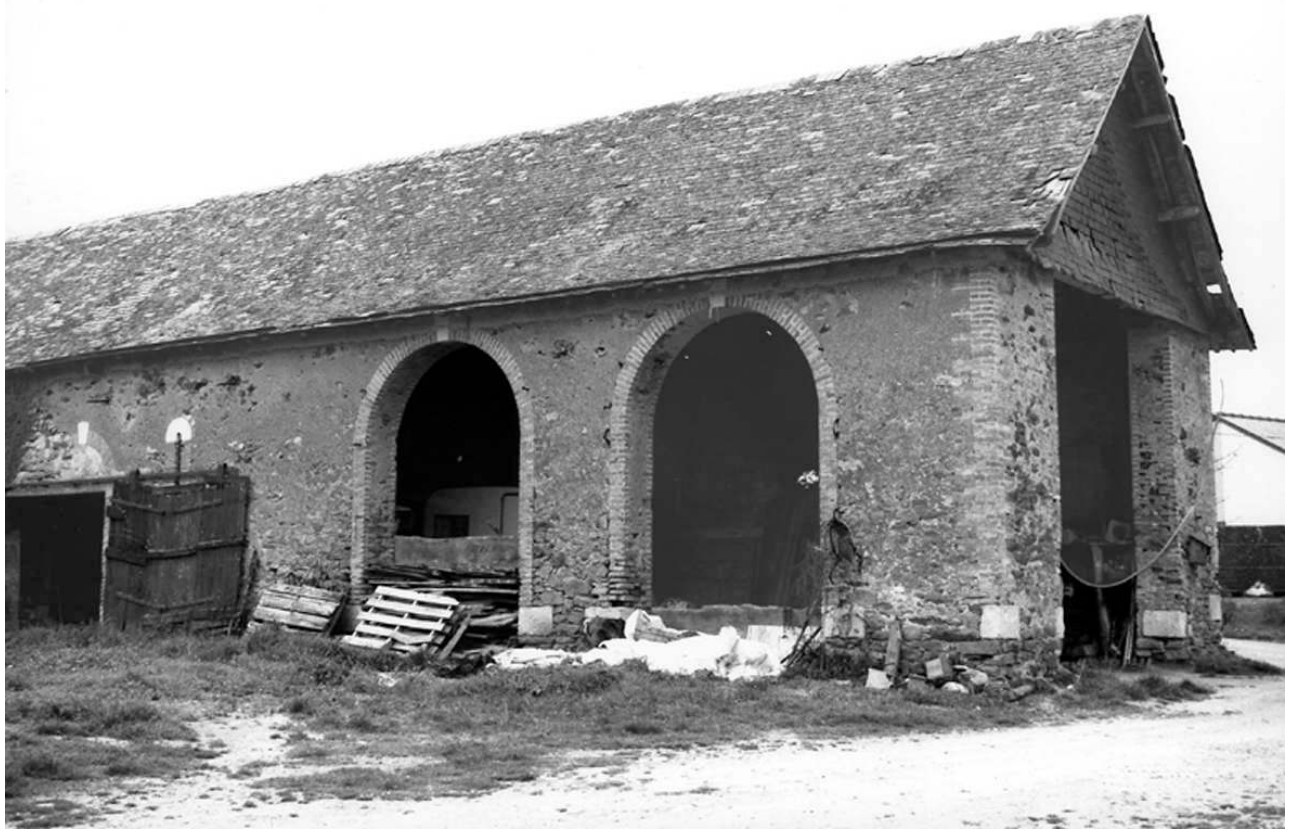

Chauvon (Le Lion-d'Angers), hangar avec larges baies encadrées de brique. Manase, Viviane (c) ADAGP/Conseil général de Maine-et-Loire, 1999. 
Le logis, couvert d'une toiture à croupes, comprend un comble en surcroît percé de jours rectangulaires en brique et tuffeau : le souci des ouvertures dans ces nouveaux bâtiments est récurrent, garantissant l'aération des zones de stockage. Ces efforts d'aménagement portent manifestement leurs fruits puisqu'en 1879, la métairie d'une superficie de 33 ha 89 ares, exploitée à colonie paritaire, rapporte 3000 francs.

Outre ces fermes héritées de leur père, les frères font de nombreuses acquisitions et développent ainsi le patrimoine familial. À titre d'exemple, La Courtière (Le Liond'Angers) est achetée par Eugène Richou (1815-1889) à Félix Raymond Duplat, baron de Monticourt en $1857^{26}$. Déjà présente sur le plan cadastral de 1809, elle est reconstruite en 1870 par Eugène en remployant le gros-œuvre de l'ancien logis transformé en étable à vaches avec « panserie ", fenil en surcroît et cave à l'arrière. Cet espace de la " panserie » participe à une meilleure organisation du travail : il permet une distribution rapide et efficace du fourrage aux animaux situés dans les étables adjacentes. Le reste des bâtiments (étable à chevaux, porcherie, remise et hangar) (fig. $\mathbf{n}^{\circ} \mathbf{1 3}$ ) a été disposé autour de la cour dotée d'un puits central sans doute plus ancien. Tout doit contribuer à un fonctionnement optimal, y compris le hangar avec deux grandes ouvertures, une côté cour et une autre sur le pignon du bâtiment, facilitant les manœuvres des machines agricoles.

Figure 13

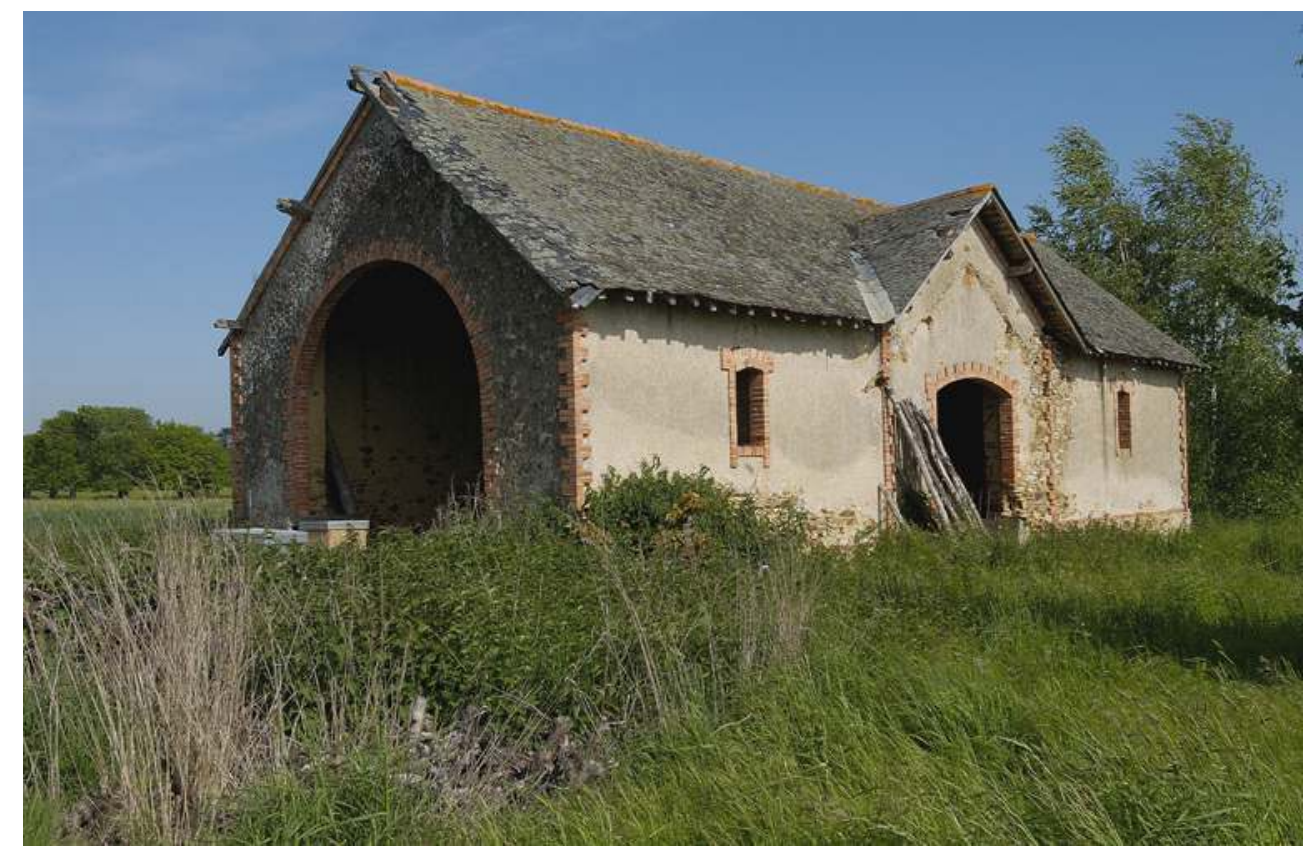

La Courtière (Le Lion-d'Angers), hangar. Rousseau, Bruno

(c) ADAGP/Conseil général de Maine-et-Loire, 2005.

27 Si les aspects pratiques sont privilégiés, le côté esthétique n'en est pas pour autant négligé : le vaste logis est ici particulièrement soigné, présentant une façade symétrique avec bandeaux et chaînages d'angle en brique (fig. $\mathbf{n}^{\circ} \mathbf{1 4}$ ). Les encadrements de baies, les oculi, également en brique, animent l'ordonnancement de la façade. La bichromie du tuffeau associé à la brique pour les aisseliers soutenant le toit est un élément supplémentaire de décor du logis. 


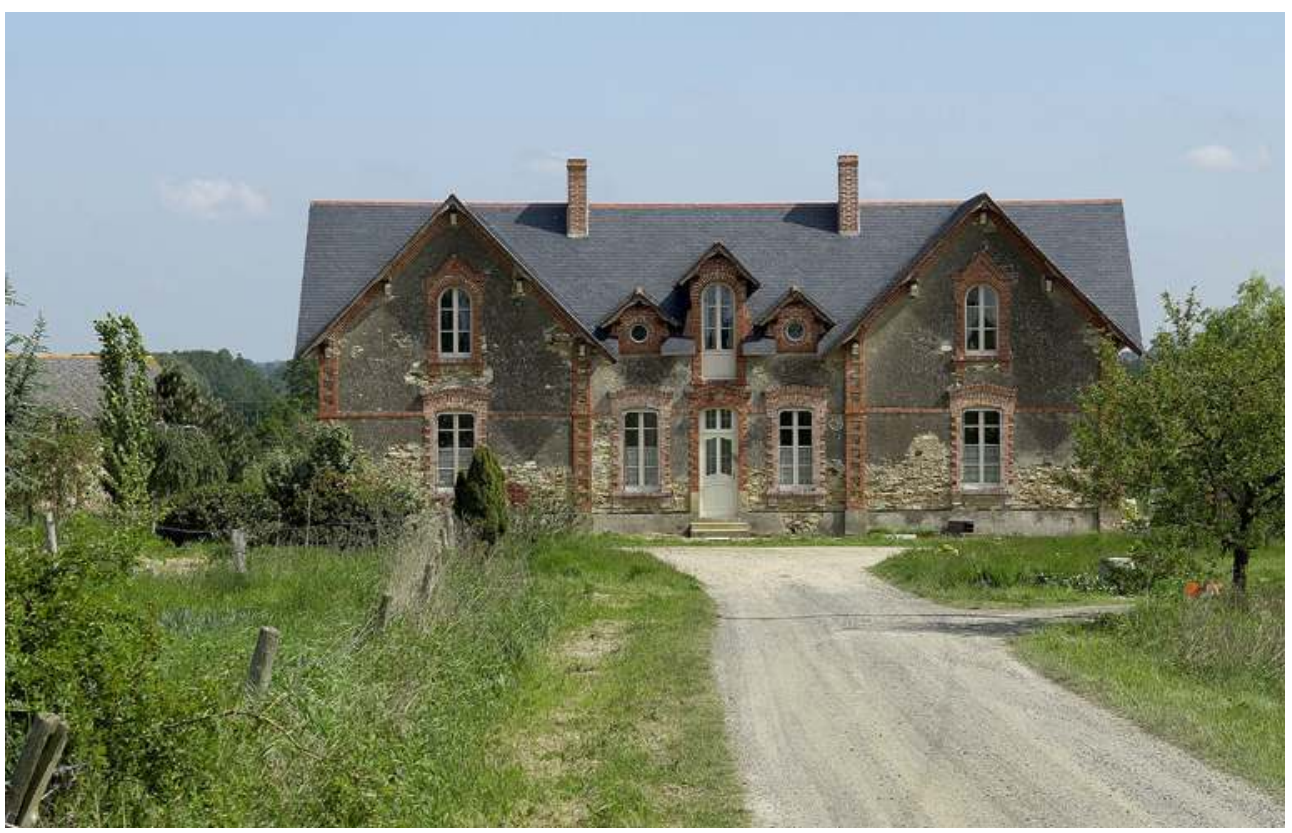

La Courtière (Le Lion-d'Angers), façade principale du logis. Rousseau, Bruno

(c) ADAGP/Conseil général de Maine-et-Loire, 2005.

Les activités des Richou s'inscrivent ainsi parfaitement dans la vague de reconstruction des fermes de la région: leurs nombreuses terres viennent compléter et accroitre la richesse générée par les moulins. L'emprise territoriale des Richou est renforcée par la présence des élégantes demeures qu'ils font édifier.

\section{L'ascension sociale des Richou}

\section{Demeures}

Dans ce vaste territoire rural, les Richou affirment leur statut social en résidant dans de fastueuses demeures dont l'architecture se distingue nettement dans la campagne du Haut-Anjou segréen. Ils rivalisent alors avec les familles nobles de la région et leurs châteaux, dont la majorité est construite ou restaurée entre 1840 et 1880 . On dénombre ainsi en Anjou 516 chantiers et 301 châteaux nouveaux au cours de cette période ${ }^{27}$.

Alors qu'une partie de la famille réside au château de la Roussière, René (II) (1774-1840) et Anne Richou achètent en 1831, à Brain-sur-Longuenée, la Maison Blanche, manoir des XVIe et XVIIe siècles, composé de deux corps de bâtiment en retour d'équerre avec dépendances ${ }^{28}$ (fig. $\left.\mathbf{n}^{\circ} \mathbf{1 5}\right)$. 


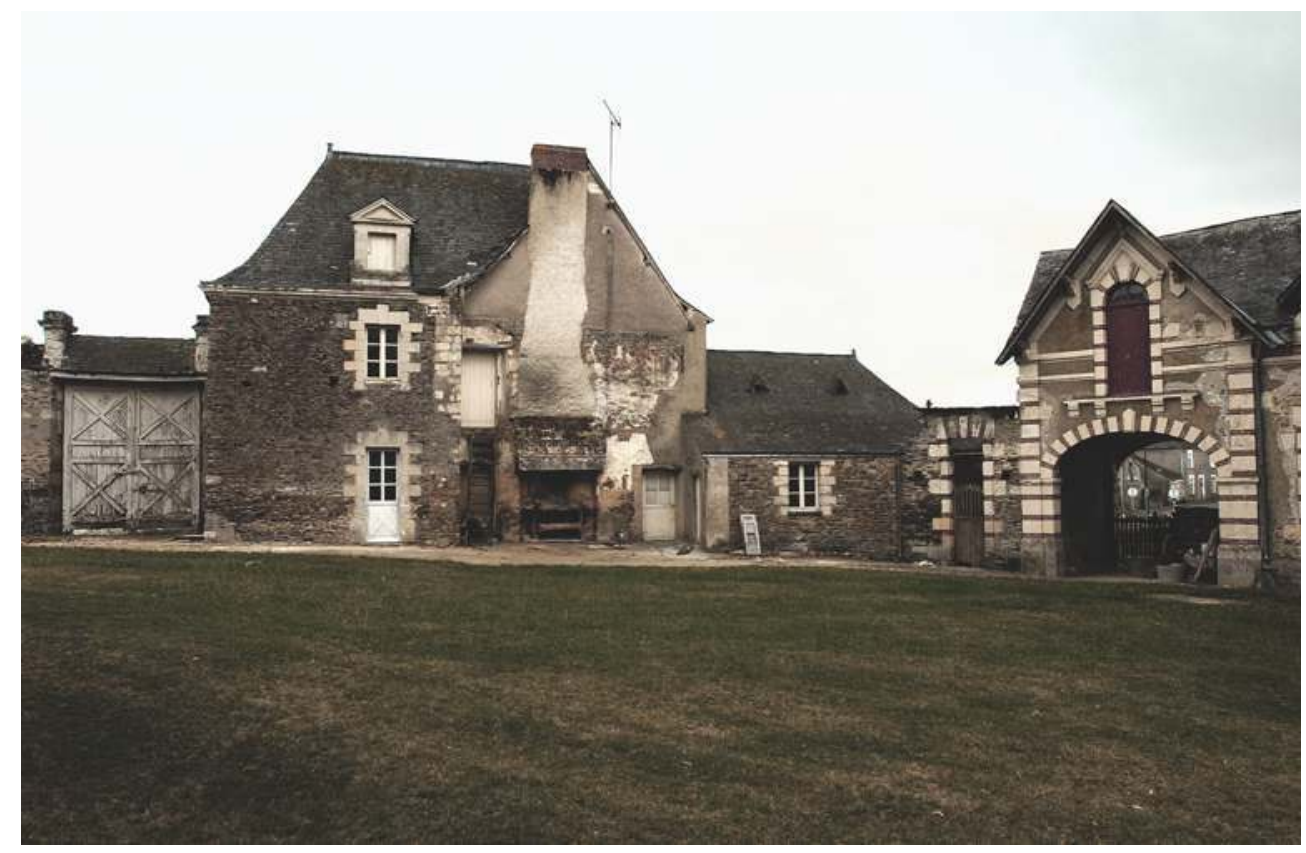

Vestiges de l'ancien manoir de la Maison Blanche (Brain-sur-Longuenée). Rousseau, Bruno (c) ADAGP/Conseil général de Maine-et-Loire, 2005.

31 À partir de 1862, Eugène Richou fait construire par l'architecte Auguste Bibard un nouveau château à proximité des anciens bâtiments en partie démolis ${ }^{29}$. Sont conservés quelques éléments, comme le pigeonnier, signe distinctif de la puissance seigneuriale que les Richou semblent vouloir reprendre à leur compte. Le nouvel édifice est solennellement béni par le curé de Brain lors d'une cérémonie à laquelle participe l'ensemble de la population en août $1864^{30}$. De plan massé, le château est composé d'un rez-de-chaussée surélevé permettant l'installation des cuisines et caves en soubassement, d'un étage carré et d'un étage de combles (fig. $\mathbf{n}^{\circ} \mathbf{1 6}$ ). 
Figure 16

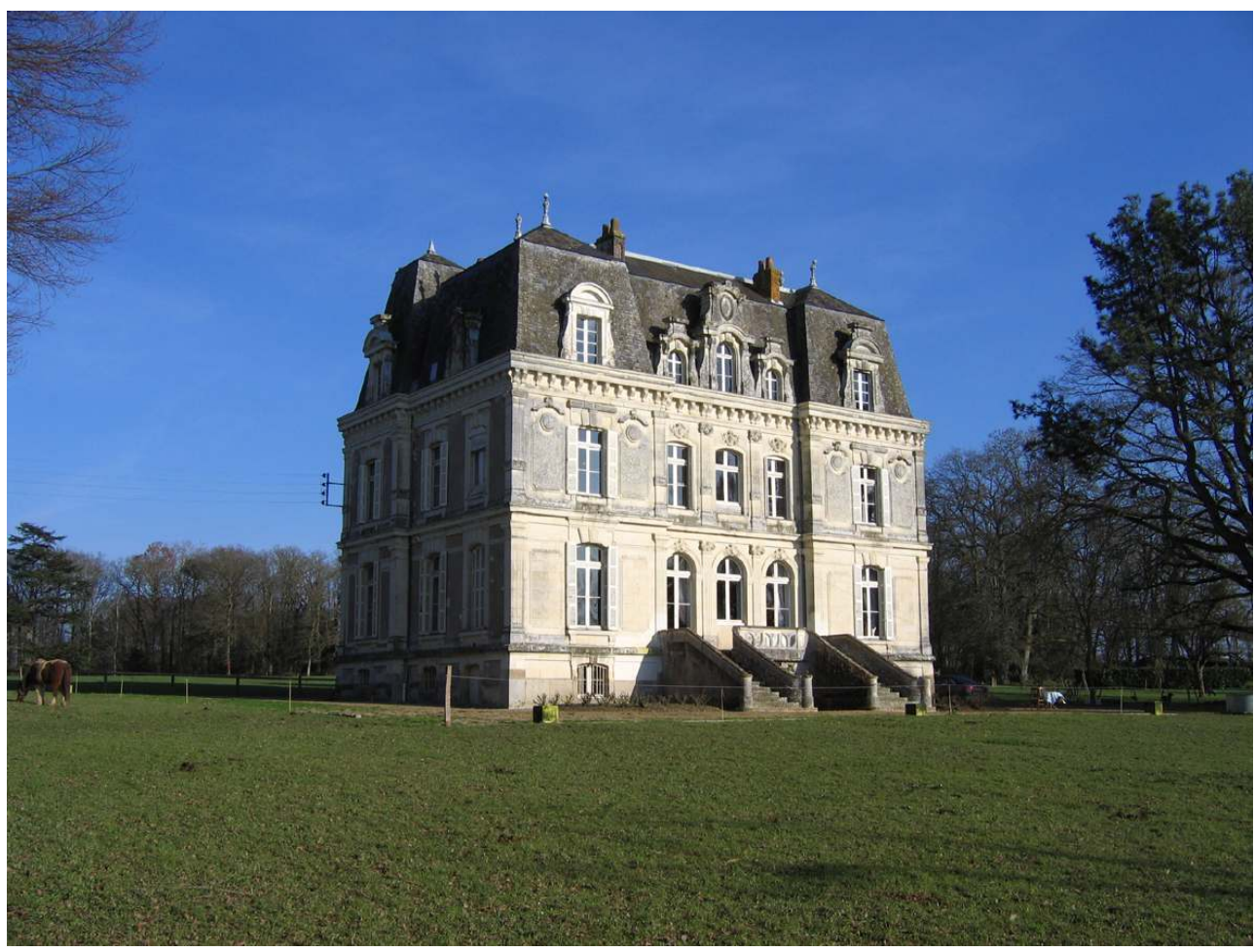

Château de la Maison Blanche (Brain-sur-Longuenée). Le Guyader, Pascal

(C) Le Guyader, Pascal, 2007.

32 Bibard n'a pas, selon son habitude, usé du style néo-Louis XIII avec parements en tuffeau et brique. Seules les hautes souches de cheminées des toitures présentent en alternance ces deux matériaux, marquant le paysage et les abords du bourg. Le décor extérieur et intérieur du château est au contraire assez sobre, tout en recherchant un certain confort avec ces cheminées disposées sous les fenêtres, offrant aussi bien le plaisir d'un bon feu que la vue sur le paysage extérieur.

Des communs sont également construits (écurie, sellerie) mais ces travaux semblent avoir été interrompus, faute d'argent peut-être. Ainsi, les anciens bâtiments, qui devaient vraisemblablement à terme être détruits, cohabitent encore avec les nouveaux. A proximité se situent les bâtiments de la métairie avec une vaste étable-grange dotée d'encadrements de baies en brique (fig. $\mathbf{n}^{\circ} \mathbf{1 7}$ ). 
Figure 17

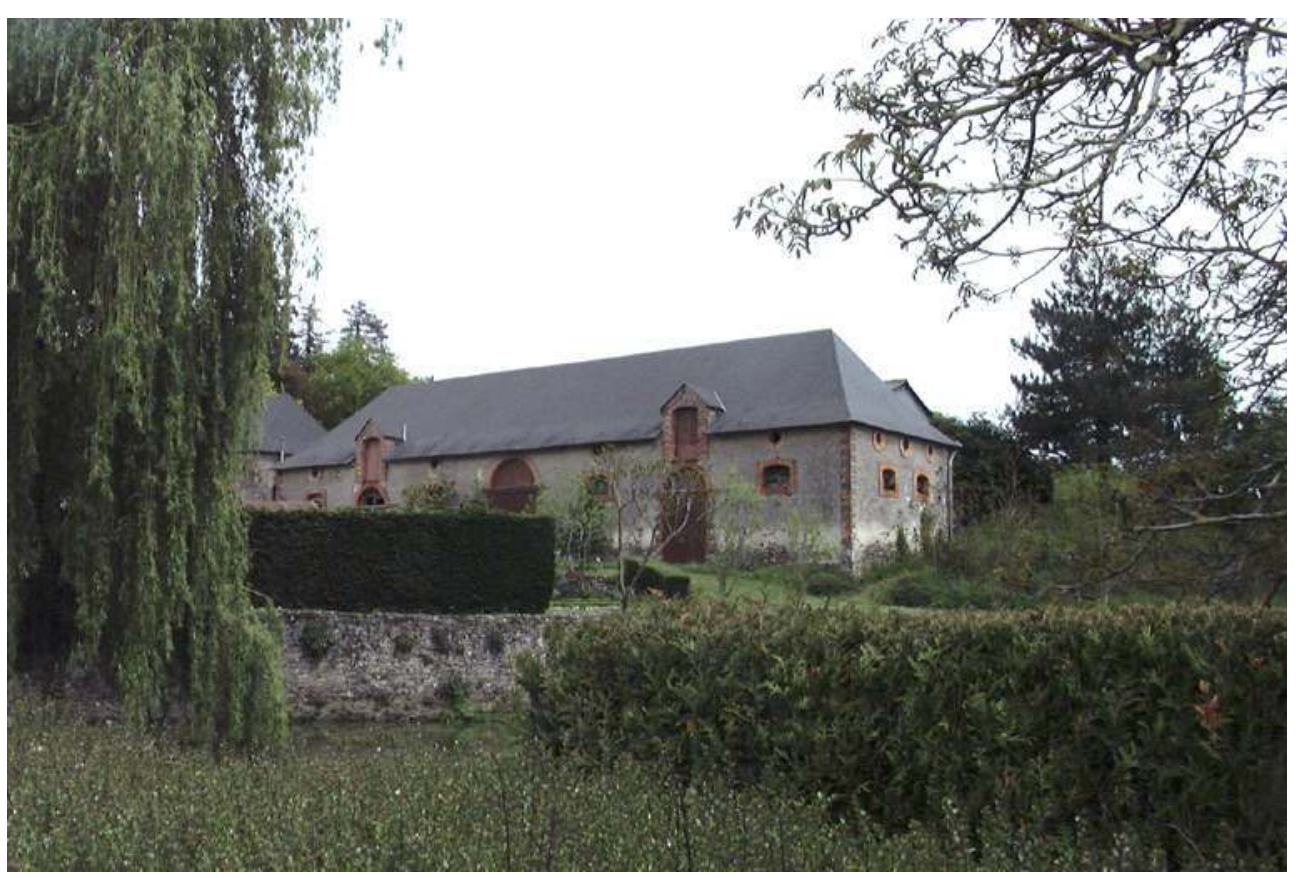

Métairie de la Maison Blanche. Steimer, Claire

(c) ADAGP/Conseil général de Maine-et-Loire, 2005.

D'autres demeures ont appartenu à la famille Richou: parmi celles-ci, le château de Cimbré à Tiercé appartient dès 1824 à la famille Boreau-Deslandes et passe par mariage à la famille Richou. C'est Arthur Richou (né en 1845), marié à Louise Boreau-Deslandes, qui fait appel à l'architecte Auguste Beignet, l'un des architectes les plus renommés d'Angers, pour transformer le château du XVIIIe siècle et lui conférer un style néogothique, en y greffant notamment de nombreuses tourelles (fig. $\mathbf{n}^{\circ} \mathbf{1 8}$ ). 


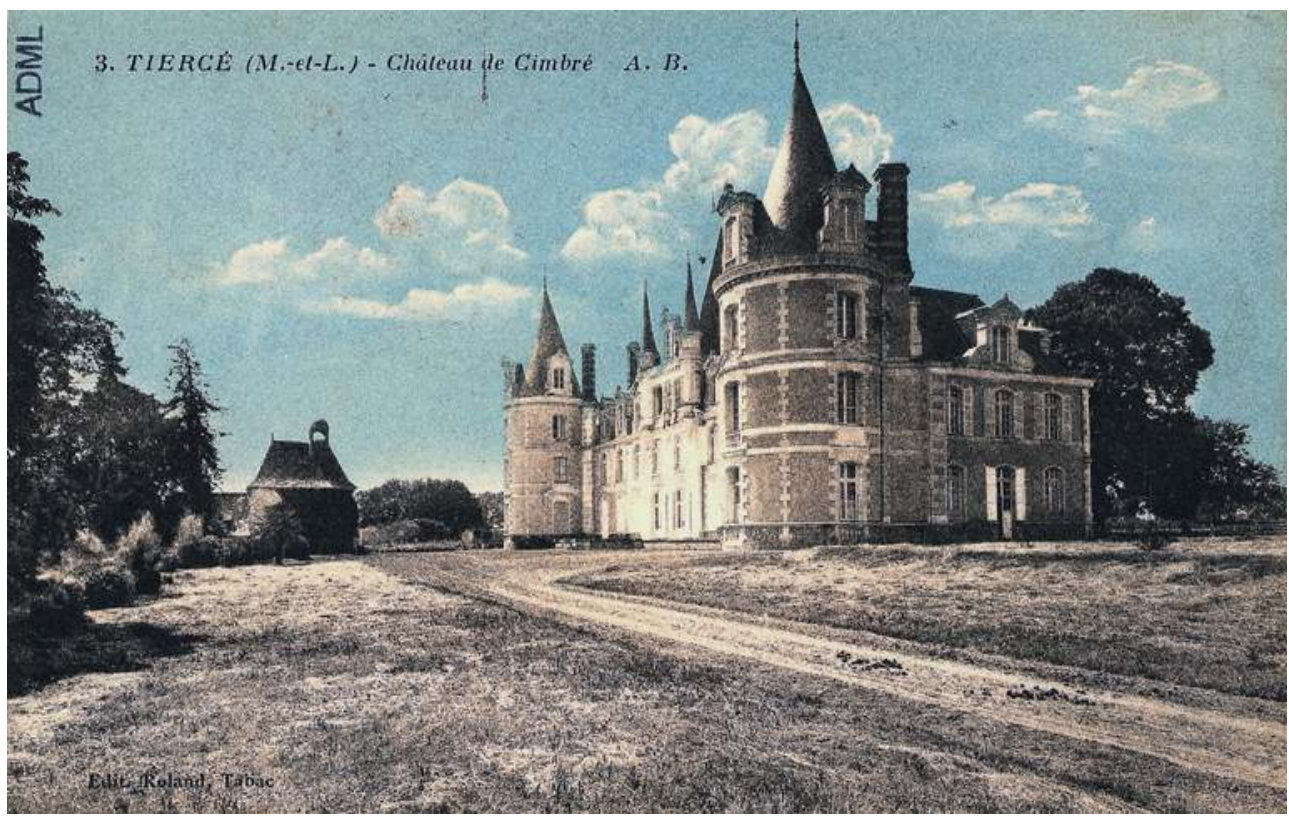

Château de Cimbré (Tiercé), carte postale. A.D. Maine-et-Loire, 6 Fi. Rousseau, Bruno

(c) Conseil général de Maine-et-Loire, 2006.

\section{Rôle dans la vie publique}

L'affirmation de cette ascension sociale se traduit par ailleurs par le rôle des Richou dans la vie publique : peu à peu, ils deviennent des notables, endossant des responsabilités locales qui participent à leur renommée. Ainsi à La Membrolle, la branche Richou-Dugré fournit bon nombre de maires pour la commune, de 1787 jusqu'en $1937^{31}$. Signalons aussi Mathurin (III) Richou, fils de Mathurin (II) Richou (1786-1859) et Perrine Houdebine, maire de Saint-Barthélémy-d'Anjou de 1868 à 1870 et de 1871 à 1881, demeurant dans cette même commune au Haut-Bois. D'autres membres de la famille Richou apparaissent dans la vie publique et politique, notamment Désiré (II) Richou (1836-1897), maire de Feneu en 1884, tout comme son fils Raymond (né en 1873) en $1904^{32}$.

À l'instar des familles nobles qui vouent quelques-uns de leurs cadets à la religion, les Richou comptent dans leurs rangs des ecclésiastiques. Le Père Alexandre Richou (1819-1897), fils de Pierre Richou et Marie Violeau dite Laroche, est formé au séminaire puis entre chez les lazaristes comme missionnaire, affecté en 1845 à leur mission de Smyrne. Autre exemple, féminin cette fois: Rose (ou Françoise) Richou, fille de Pierre Richou et Renée Soreau, religieuse sécularisée à la Révolution, s'installe à La Membrolle pour se dévouer aux malades et à l'instruction des enfants. En 1820, elle lègue à la Congrégation de la Sagesse une maison et divers immeubles dont une ferme dénommée la Babinière, à charge de fonder dans la paroisse une maison d'instruction et de charité qui serait dirigée à perpétuité par trois filles de la Sagesse. Cette école fonctionnera jusqu'en $1913^{33}$ et, aujourd'hui encore, une rue de la Membrolle porte le nom de Rose Richou.

La famille se distingue également par des signes de libéralité religieuse : lorsque Eugène Richou achète la ferme de la Saulaie à Brain-sur-Longuenée, il fait dresser une croix au carrefour à proximité ${ }^{34}$. En revanche, on ne remarque pas, dans les parcs de leurs 
demeures, de chapelle privée qui pourrait témoigner de leur attachement à une pratique religieuse personnelle.

La sphère d'influence de la famille s'étend à de nombreuses activités dont témoignent les annuaires statistiques de Maine-et-Loire ${ }^{35}$ : banque, extraction (ardoises, bois de chauffage, fers), culture (chanvres, filasses, huiles et denrées du pays), commerces (boulangerie, charcuterie), travaux des ponts et chaussées... Joseph Richou (1796-1859), issu de la branche Richou-Guilleux, fils de Joseph Richou et Marie-Victoire Marais, est architecte. Auteur de la reconstruction en 1856 du chœur et d'une partie de la nef de l'église Saint-Jacques à Angers, son nom apparaît aussi dans les archives de la commune de Brain-sur-Longuenée, où il réalise des plans pour la mairie-école. Il n'est pas le seul architecte de la famille : Auguste Beignet est dans un premier temps marié à Rose Richou (1852-1886), puis à la mort de celle-ci, il se remarie avec une de ses parentes, Marie Thibault, arrière-petite-fille de Marie-Anne Richou et Nicolas Joubert. Ce rapprochement matrimonial explique certainement l'intervention de Beignet au château de Cimbré à Tiercé.

Figure 19

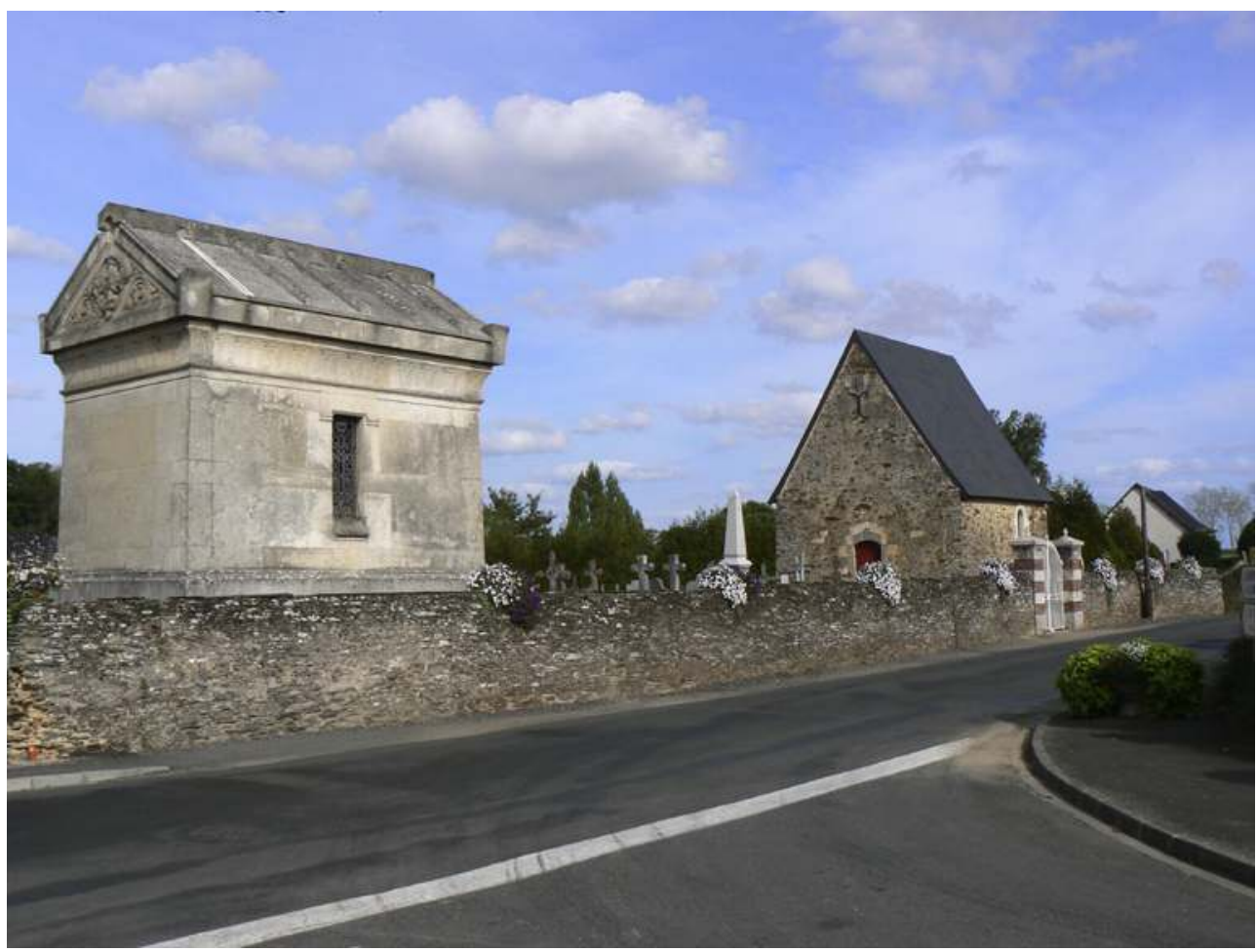

Chapelle funéraire des Richou, face à celle des Mauvif de Montergon (1640) dans le cimetière de Brain-sur-Longuenée. Steimer, Claire

(c) Conseil général de Maine-et-Loire, 2006.

Les Richou acquièrent ainsi une place à part et s'implantent fortement dans la vie locale. Ils y côtoient alors les membres des familles nobles : ainsi le 9 juin 1851, le curé de Brainsur-Longuenée déjeune «chez M. et Mme Richou en société de M.M. Mauvif de Montergon et Poidevin, et de M. et Mme Richou d'Angers, marchands de fer et de leurs enfants qui arrivèrent pendant le repas $\|^{36}$. Dans le cimetière de Brain-sur-Longuenée, la chapelle funéraire des Richou, construite par Beignet, s'élève d'ailleurs aux côtés de celle des Mauvif de Montergon, d'égal à égal (fig. $\mathbf{n}^{\circ}$ 19). 


\section{Alliances matrimoniales}

Il existe d'autres similitudes avec les mœurs et coutumes des grandes familles nobles, à savoir la stratégie matrimoniale. L'endogamie familiale et géographique semble la règle afin d'éviter l'émiettement du patrimoine et de favoriser la continuité de leurs activités de génération en génération. Les mariages entre membres de la famille sont fréquents : citons par exemple René (II) qui épouse en 1807 sa cousine Anne Richou, fille de Mathurin (I) Richou, ou encore Alexandre (I) qui épouse en 1834 sa cousine Claire Raffray, fille de Rose Richou et Jacques Raffray.

41 Les alliances matrimoniales ont aussi contribué à l'ascension sociale de la famille Richou qui a su s'allier à d'illustres familles. Jeanne-Martine Richou, fille de René (I) Richou épouse Pierre-François Besnard : leur fils François est à l'origine, dans les années 1840, de la célèbre industrie de corderie d'Angers, reprise par son gendre Julien Bessonneau, dont le nom est encore lié à cette activité industrielle angevine. Julien Bessonneau est doublement lié à la famille Richou, puisqu'il est le fils de Jeanne-Françoise Besnard, fille de Jeanne-Martine Richou, mariée à Julien-Pierre-Maurille Bessonneau. Autre exemple d'union prestigieuse : le banquier Désiré (II) Richou épouse en 1866 Marguerite Segris, fille du futur ministre Emile Segris (fig. $\mathbf{n}^{\circ} \mathbf{2 0}$ ).

Figure 20

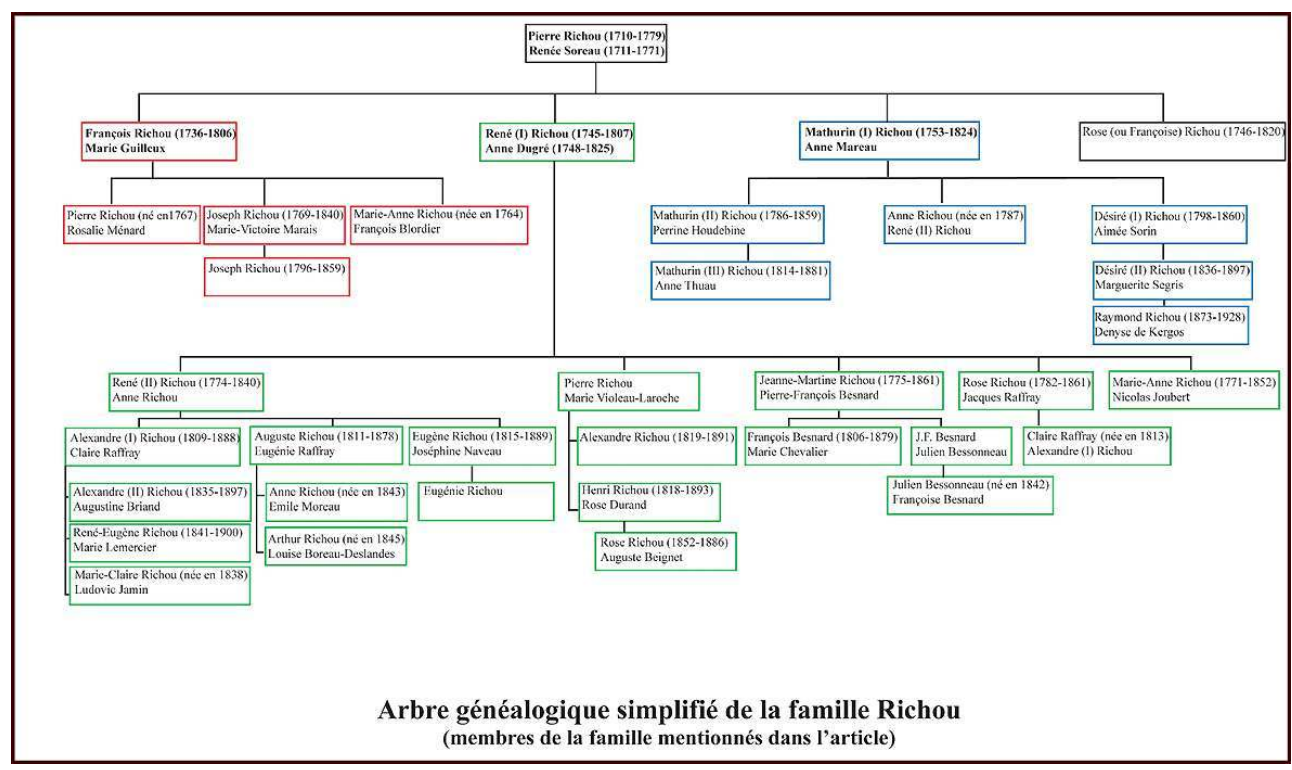

Arbre généalogique simplifié réalisé à partir de celui conservé aux Archives de Maine-et-Loire (1 F 13). Steimer, Claire

(c) Steimer, Claire, 2006.

Leurs multiples activités, leur style de vie et leurs relations sociales montrent l'évolution des Richou dans la société du XIXe siècle. Leur réputation s'élargit encore avec l'activité bancaire dont ils sont les précurseurs à Angers. 


\section{L'activité bancaire}

43 La branche Richou-Mareau, avec notamment Désiré (I) Richou (1798-1860), fait évoluer les activités de la famille et ouvre l'ère de la banque Richou. Non seulement influents sur le monde agricole et industriel, ils sont aussi des acteurs incontournables de la société urbaine. Les plus anciens banquiers d'Angers sont les sieurs Philippe Rogeron et Louis et Frédéric Gaultier, déjà associés pour exercer cette fonction avant 1810. Mais dès 1822, Philippe Rogeron s'associe à Désiré (I) Richou pour continuer ses affaires et en 1827, celuici se retire pour fonder une banque personnelle : il apparaît ainsi en 1830 dans l'annuaire départemental comme l'un des trois banquiers angevins. Tout au long du XIXe siècle, on trouve mention des Richou dans le monde de la banque: en 1850, au moment même où s'établit à Angers la succursale de la Banque de France et la banque Bigot-Bougère, la ville possède sept établissements de crédit dont Richou et Raguin, rue Boisnet et dix ans plus tard, neuf banquiers - dont Veuve Richou et fils - coexistent sur la place d'Angers, indépendamment de la Banque de France.

La banque Richou était installée 23-25 rue Boisnet, ce bâtiment abritant également un immeuble de rapport et le logement de la famille ${ }^{37}$ (fig. $\mathbf{n}^{\circ} \mathbf{2 1}$ ). En partie du XVIIe siècle, il est remanié et agrandi entre 1848 et 1851.

Figure 21

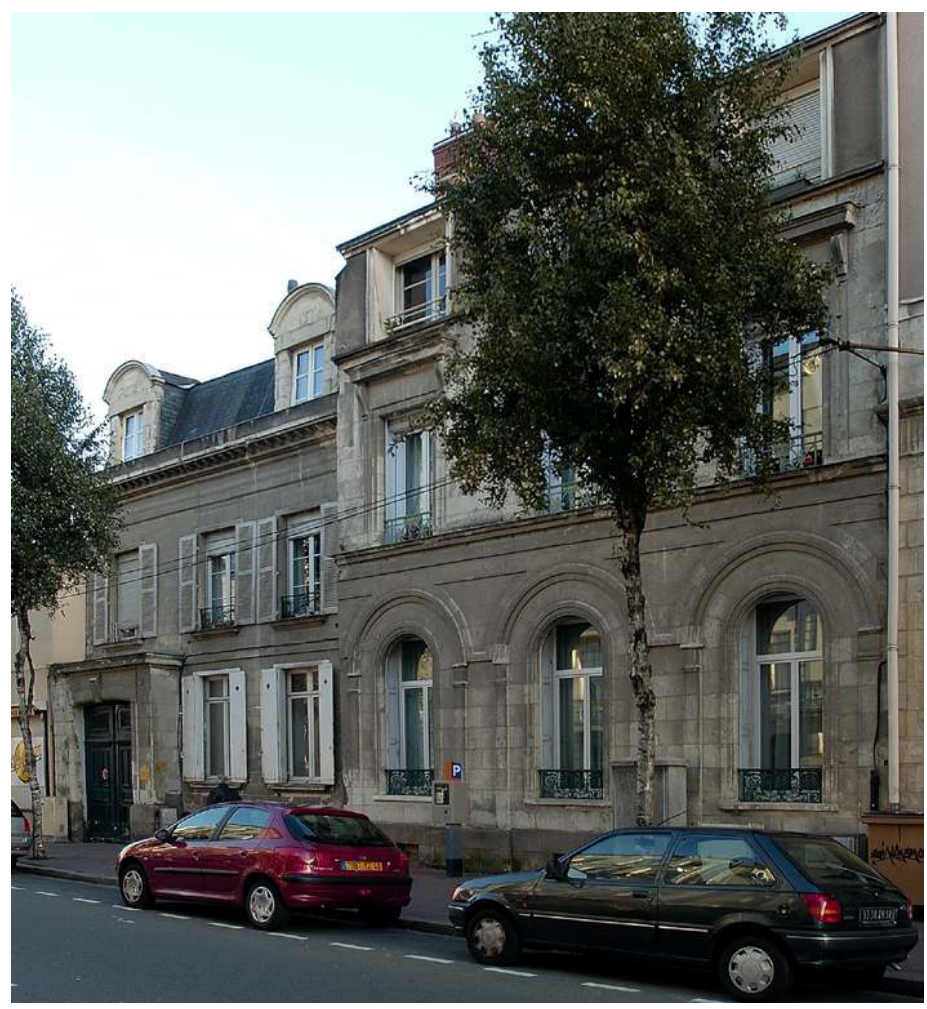

Banque et logement des Richou, rue Boisnet à Angers. Rousseau, Bruno (c) Conseil général de Maine-et-Loire, 2006.

Des bureaux de la banque, aujourd'hui détruits, existaient aussi place de Lorraine et on retrouve boulevard Foch un bâtiment de style néoclassique qui semble bien être celui construit en 1867 par Désiré (II) Richou ${ }^{38}$ (fig. $\mathbf{n}^{\circ}$ 22). 


\section{Figure 22}

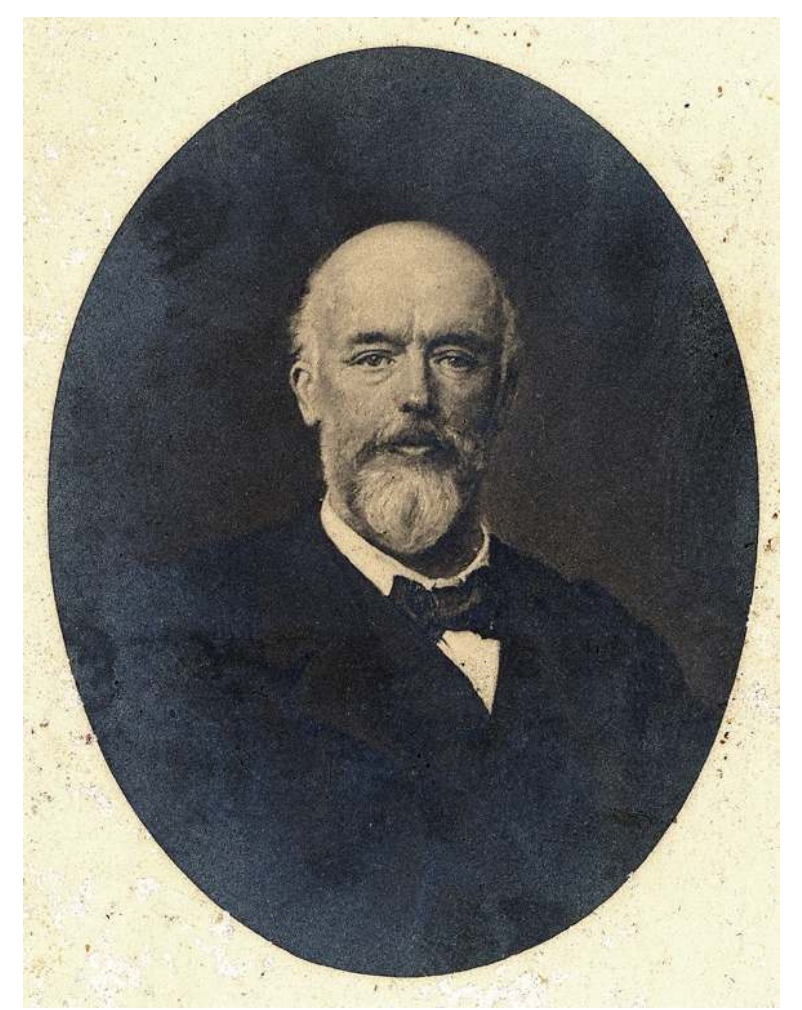

Portrait photographié de Désiré Richou. A.D. Maine-et-Loire, 1 F 13. Rousseau, Bruno (C) Conseil général de Maine-et-Loire, 2006.

La banque Richou possédant par ailleurs des succursales dans d'autres villes comme Saumur, l'implantion urbaine de la famille ne se limitait donc pas à Angers et leur influence rayonnait largement en Anjou. Propriétaires de demeures à la campagne, les Richou possédaient aussi bien souvent un appartement à Angers. Ainsi, Raymond Richou, fils de Désiré (II) et Marguerite Segris, qui hérite de la banque de son grand-père et de son père, habite au 41 boulevard de Saumur à Angers mais aussi dans l'élégante demeure des Grifferais à Savennières (fig. $\mathbf{n}^{\circ}$ 23). 


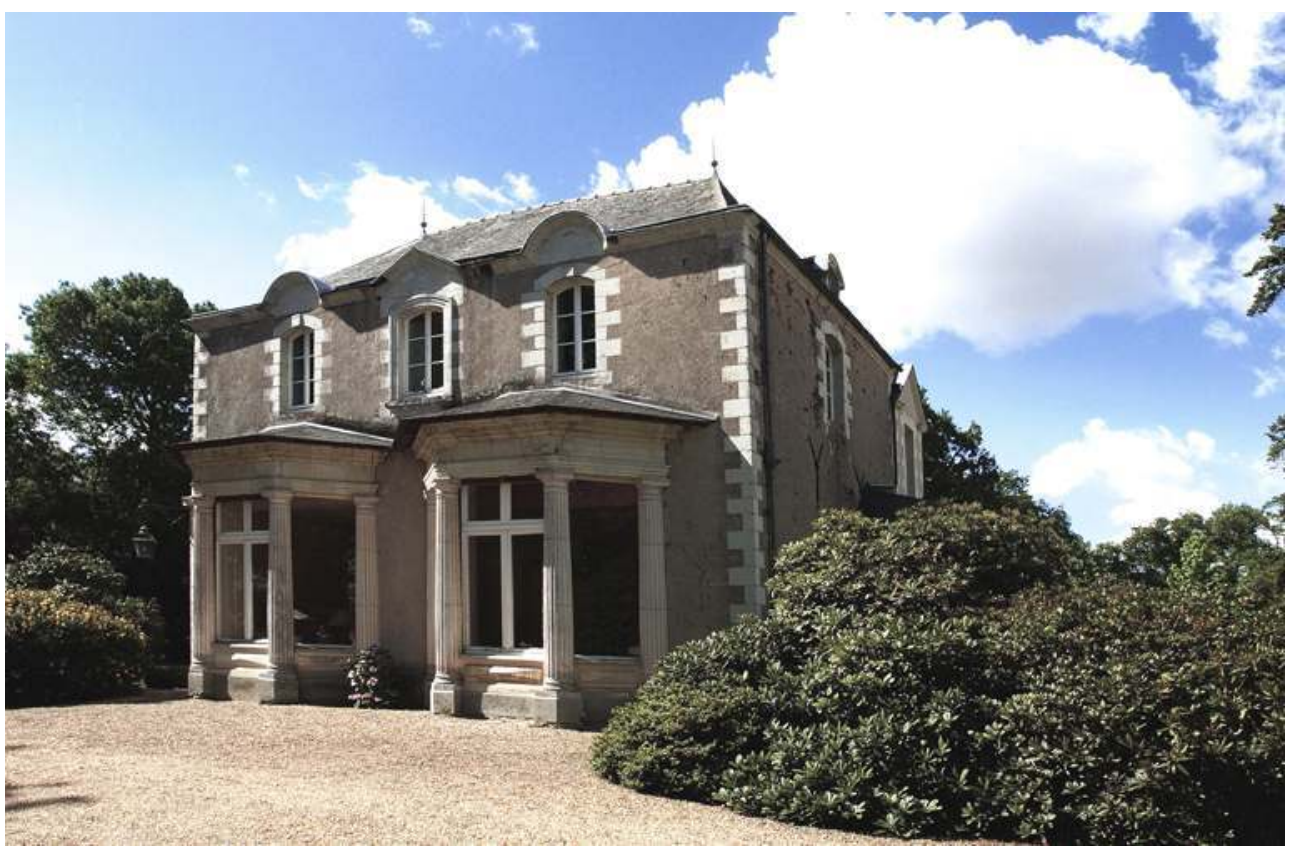

Les Grifferais à Savennières. Rousseau, Bruno

(c) ADAGP/Conseil général de Maine-et-Loire, 2005.

Ce membre de la famille Richou ne faillit pas aux caractéristiques évoquées ci-dessus et s'inscrit parfaitement dans la tradition familiale: sa femme, Denyse de Kergos, issue d'une famille noble, lui apporte par mariage la propriété de Savennières. Responsable de charges importantes et honorifiques, il est nommé vice-consul de Sa Majesté britannique à Angers en 1912 et reçoit la Légion d'honneur en 1920. 
Figure 24

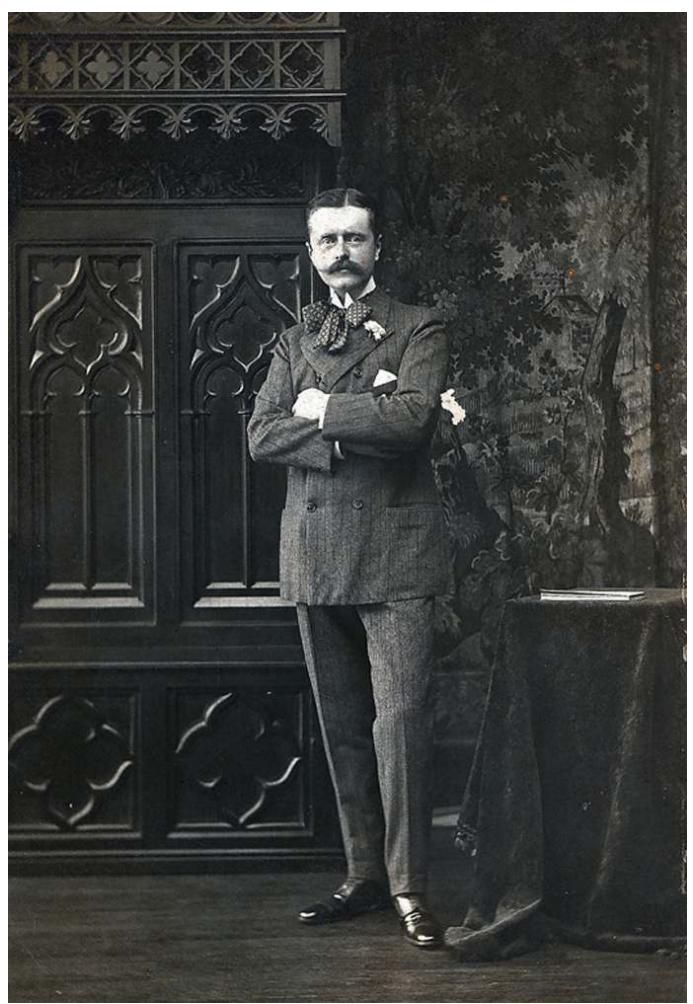

Portrait photographié de Raymond Richou. A.D. Maine-et-Loire 1 F 13. Rousseau, Bruno (c) Conseil général de Maine-et-Loire, 2006.

Banquier comme son père et son grand-père depuis 1897 , (fig. $\mathbf{n}^{\circ}$ 24) il est également maire de Feneu de 1904 à 1919. C'est avec lui que va s'opérer la liquidation de la banque Richou, cédée à la Banque nationale de Crédit en avril 1914, dont il est alors nommé directeur régional ${ }^{39}$. Une lettre du 31 mars 1916 adressée par Raymond Richou à ses enfants montre que, loin de mettre fin à la richesse des Richou, cette liquidation à la veille de la Première Guerre mondiale a été plutôt bénéfique à la famille. Il a pu ainsi sauver une partie de la fortune laissée par son père ${ }^{40}$.

49 Malgré d'inévitables aléas, les Richou ont su, de la fin du XVIIIe siècle au début du XXe siècle, gérer au mieux leurs biens, diversifier leurs activités en investissant des domainesclés de l'économie régionale tels que la meunerie, l'agriculture et la banque. Leur ascension sociale doit beaucoup aux innovations qu'ils ont adoptées assez rapidement : la fondation en 1827 d'une des premières banques angevines, le passage de la meunerie traditionnelle à l'ère des minoteries avec en 1847 l'usine de La Roussière et enfin la construction, dans les années 1860-1880 surtout, de nombreuses fermes modernes et d'une qualité architecturale indéniable, centres performants de terres agricoles productives. Leur parfaite intégration dans le monde de l'époque tient aussi à leur mode de vie, commun aux notables du XIXe siècle, imitant plus ou moins le modèle aristocratique.

Par leur réussite sociale, politique et économique, des familles ont ainsi façonné nos campagnes et nos villes en y édifiant nombre d'édifices qui témoignent de riches histoires familiales et, plus largement, de l'histoire de tout un territoire ${ }^{41}$. 


\section{NOTES}

1. Inventaire topographique en cours (Viviane Manase, Claire Steimer) et étude menée en particulier à partir des registres d'hypothèques (A.D. Maine-et-Loire, série $4 \mathrm{Q}$, transcriptions d'actes translatifs de propriétés immobilières entre vifs).

2. A.D. Maine-et-Loire, fonds Segris, $1 \mathrm{~F}$ 13. Les références généalogiques que nous avons utilisées pour cette recherche proviennent de ces documents. Lorsque plusieurs individus portent le même prénom, nous leur attribuons un numéro (I, II, III...) afin d'éviter toute confusion.

3. A.D. Maine-et-Loire, 1 B $51 \mathrm{f}^{\circ} 169 \mathrm{v}^{\circ}$.

4. De ce couple Richou-Soreau sont issues les personnes mentionnées tout au long de l'article, et en premier lieu, leurs trois fils, François, René (I) et Mathurin (I), à l'origine des trois grandes branches (Richou-Guilleux, Richou-Dugré et Richou-Mareau) que nous distinguons dans notre étude.

5. Sur la connaissance des moulins en Anjou: CUSSONNEAU, Christian. Moulins d'Anjou. [Nantes] : Inventaire général, ADIG, 1991 (coll. Images du Patrimoine, $\mathrm{n}^{\circ}$ 90).

6. A.D. Maine-et-Loire, registre des hypothèques, 4 Q 2937 : transcription de ces aveux du 15e siècle.

7. A.D. Maine-et-Loire, matrices cadastrales, augmentations et diminutions : démolition du moulin en 1845 et reconstruction en 1846 (parcelle B271).

8. PORT, Célestin. Dictionnaire historique, géographique et biographique de Maine-et-Loire, édition revue et augmentée, t. 3, 1989, p. 530.

9. A.D. Maine-et-Loire, matrices cadastrales, augmentations et diminutions : augmentation de construction d'une maison en 1869 (parcelle B272), du moulin en 1878 (parcelle B271).

10. A.D. Maine-et-Loire, registre des hypothèques, $4 \mathrm{Q} 17587$.

11. A.D. Maine-et-Loire, $126 \mathrm{~S}$ 12, procès-verbal de visite, 8 juin 1875.

12. A.D. Maine-et-Loire, $126 \mathrm{~S}$ 12, rapport de l'ingénieur ordinaire, 8 novembre 1883.

13. A.D. Maine-et-Loire, matrices cadastrales, augmentations et diminutions : parcelle B271, moulin de la Roussière incendié en 1919.

14. PORT, Célestin. Dictionnaire historique, géographique et biographique de Maine-et-Loire , édition revue et augmentée, t. 3, 1989, p. 350. Voir aussi les matrices cadastrales, augmentations et diminutions (B346 Le Rocher, maison construction nouvelle en 1854).

15. CUSSONNEAU, Christian. Vocabulaire de la meunerie en Anjou, à travers les sources d'archives (XIIe-XIXe siècle). Les Cahiers de l'A.M.A., Association des Amis des Moulins de

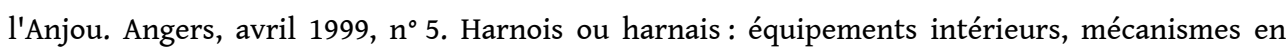
général.

16. A.D. Maine-et-Loire, registre des hypothèques $4 \mathrm{Q} 17398$.

17. A.D. Maine-et-Loire, registre des hypothèques $4 \mathrm{Q} 17422$.

18. A.D. Maine-et-Loire, registre des hypothèques, $4 \mathrm{Q} 17369$.

19. A.D. Maine-et-Loire, registre des hypothèques, $4 Q 2604$ et 2071.

20. A.D. Maine-et-Loire, registre des hypothèques, $4 \mathrm{Q} 17351$.

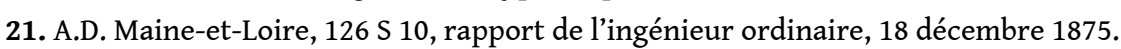

22. CUSSONNEAU, Christian. Moulins d'Anjou. [Nantes]: Inventaire général, ADIG, 1991 (coll. Images du Patrimoine, $\mathrm{n}^{\circ}$ 90).

23. A.D. Maine-et-Loire, registre de l'Enregistrement, 4 Q 17387.

24. Terme local désignant la zone de stockage du fourrage du jour pour les animaux.

25. A.D. Maine-et-Loire, Enregistrement, bureau du Lion-d'Angers, 3 Q 9826. 
26. A.D. Maine-et-Loire, registre des hypothèques, $4 \mathrm{Q} 17467$.

27. LEICHER, Anna. Le château en Anjou entre 1840 et 1880. Thèse de doctorat. Lyon, Université Lumière-Lyon II, 1999, dactyl.

28. A.D. Maine-et-Loire, registre des hypothèques, 4 Q 17397, adjudication volontaire et définitive du 13 avril 1831.

29. Manuscrit du curé Gourdon conservé à la mairie de Brain-sur-Longuenée : « Aujourd'hui jeudi huitième jour du mois de mai 1862, pour répondre à l'invitation de Monsieur et Madame Eugène Richou, je me suis transporté sur le lieu où se construit leur maison de la Maison Blanche et j'ai frappé avec eux la première pierre de cette dite maison, M. Bibard d'Angers étant l'architecte, et le sieur Jérémie David du Lion l'entrepreneur pour la bâtisse ».

30. Mention du curé Gourdon.

31. PORT, Célestin. Edition revue et augmentée, t. 2, 2004, p. 262 : René Richou, syndic en 1787, puis René Richou en 1801, René Richou fils en 1826, Alexandre Richou en 1846, Alexandre Richou en 1856, René Richou en 1884, Louis-Jamin Richou en 1900 et Louis Jamin en 1937.

32. PORT, Célestin. Edition revue et augmentée, t. 2, 1978, p. 139.

33. PORT, Célestin. Edition revue et augmentée, t. 2, 2004, p. 262.

34. Manuscrit du curé Gourdon : «Depuis bien longtemps la croix du carrefour de la Saulais sur le chemin de Grez-Neuville était dans un triste état de mutilation. La métairie de la Saulais ayant été acquise par M. et Mme Eugène Richou, les nouveaux propriétaires de cette ferme songèrent à élever une nouvelle croix sur le dit carrefour de la Saulais ».

35. A.D. Maine-et-Loire, PER 7 : annuaires dépouillés de 1809 à 1899.

36. Manuscrit du curé Gourdon.

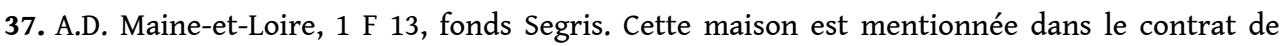
mariage de Désiré (II) Richou et Marguerite Segris en date du 4 juin 1866 (Me Hasselmann, Angers) : « une maison située à Angers, rue Boisnet et rue Basse du Mail actuellement occupée par Mme veuve Richou et son fils et par les bureaux de leur maison de banque. Telle que cette maison a été attribuée à Monsieur Richou, futur époux au $2^{\mathrm{e}}$ lot d'un partage fait entre lui, Mme Aimée Richou, épouse de M. Edme Gustave Batereau et Mme Louise Urbaine Richou, épouse de M. Edmond Marie Lorieux, ses deux sœurs, acte passé devant Me Pelou à Angers le 15 juin 1862, contenant aussi donation par Mme veuve Richou à ses trois enfants susnommés ".

38. A.D. Maine-et-Loire, annuaires statistiques de Maine-et-Loire. Communications orales de 0. Biguet et D. Letellier.

39. LEVRON, Jacques. Crédit de l'Ouest centenaire du siège social d'Angers 1850-1950, Historique, le Crédit et la Banque en Anjou (XVIe siècle à nos jours). Tours, 1950.

40. A.D. Maine-et-Loire, $1 \mathrm{~F}$ 13. Ce document mentionne la somme de quatre millions en immeubles, valeurs mobilières et créances.

41. Je tiens à remercier Christian Gasnier, A.D. Maine-et-Loire, Dominique Letellier et Olivier Biguet, Service de l'Inventaire, Ville d'Angers. La relecture a été assurée par Thierry Pelloquet, Ronan Durandière, service départemental de l'Inventaire de Maine-et-Loire; Christian Cussonneau, service régional de l'Inventaire des Pays de la Loire. 


\section{RÉSUMÉS}

L'étude de la famille Richou de la fin du XVIIIe siècle au début du XXe siècle en Maine-et-Loire s'appuie sur ses propriétés encore conservées: moulins, fermes, châteaux, hôtels particuliers, banque. L'évocation de certains membres de la famille permet de montrer l'évolution d'une famille bourgeoise au cours du XIXe siècle, marqué par les révolutions agricoles et industrielles. Les Richou se sont ainsi illustrés tout d'abord dans la meunerie, participant activement à la modernisation de cette activité et au développement des minoteries modernes. Ils ont également conforté leur situation avec l'achat de nombreuses terres et la construction ou le réaménagement de fermes répondant aux nouveaux critères productifs et hygiénistes de la 2e moitié du XIXe siècle. Enfin, ils sont à l'origine d'une des premières banques angevines. A travers cette famille, ce sont donc les grands pôles d'activité du XIXe siècle qui sont étudiés (agriculture, industrie, commerce) ainsi que leurs témoins architecturaux préservés.

This study of the Richou family, from the end of the 18th century up to the beginning of the 20th, is based on the analysis of the family's properties which still exist in the Maine-et-Loire department: mills, farms, chateaux, town houses and bank. A closer examination of certain members of the family illustrates the evolution of a bourgeois dynasty during the 19th century characterised by its agricultural and industrial revolutions. The Richous first came to the fore as millers, actively participating in the modernisation of milling techniques and constructing modern industrial flourmills. They consolidated their wealth by land purchases and the construction or modernisation of farm buildings corresponding with the new criteria of agricultural production and hygiene during the second half of the 19th century. Finally, the Richous were at the origins of one of the first banking houses in the Anjou region. The family's history thus offers material for the study of the main poles of activity during the 19th century, agriculture, industry, commerce, and for the surviving architectural manifestations of these activities.

\section{INDEX}

Mots-clés : architecte, banque, château, famille, ferme, métairie, meunerie, minoterie, moulin, révolution agricole, révolution industrielle, XIXe siècle

Keywords : 19th century, agricultural revolution, architect, bank, family, farm, flour milling, industrial mill, industrial revolution, mill, The Richou family

\section{AUTEURS}

\section{CLAIRE STEIMER}

Conservateur du patrimoine. Conseil général de Maine-et-Loire. c.steimer@cg49.fr 


\section{VIVIANE MANASE}

Conservateur du patrimoine. Service chargé de l'inventaire général du patrimoine culturel, Région Haute-Normandie. viviane.manase@cr-haute-normandie.fr 